\title{
Moderate deviations principles for the kernel estimator of nonrandom regression functions
}

\author{
Abdelkader Mokkadem ${ }^{1, *}$ and Mariane Pelletier ${ }^{1}$ \\ ${ }^{1}$ Laboratoire de Mathématiques de Versailles, UVSQ, CNRS, Université Paris-Saclay, 78035 Ver- \\ sailles
}

Received April 5, 2016; Accepted November 26, 2016

Copyright (C) 2016, Afrika Statistika and Statistics and Probability African Society (SPAS). All rights reserved

\begin{abstract}
The aim of this paper is to provide pointwise and uniform moderate deviations principles for the kernel estimator of a nonrandom regression function. Moreover, we give an application of these moderate deviations principles to the construction of confidence regions for the regression function.

Résumé. L'objectif de cet article est de donner les principes de déviations modérées, ponctuels et uniformes, satisfaits par l'estimateur à noyau d'une fonction de régression déterministe. De plus, nous donnons une application de ces principes de déviations modérées à la construction de régions de confiance pour la fonction de régression.
\end{abstract}

Key words: Moderate deviations principles, confidence interval, confidence band. AMS 2010 Mathematics Subject Classification : 62G08, 62G15, 62G20.

\section{Introduction}

We consider the following regression model

$$
Y_{i, n}=\mu\left(t_{i, n}\right)+\varepsilon_{i, n}, i=1, \ldots, n,
$$

where $\mu$ is an unknown real function defined on the interval $[0,1]$, the errors $\varepsilon_{i, n}$ form a triangular array of independent and identically distributed random variables with expectation zero and finite variance $\sigma^{2}$, and $\left(t_{1, n}, t_{2, n}, \ldots, t_{n, n}\right)$ is the sampling of the interval $[0,1]$ defined by $t_{i, n}=(i-1) / n$. This assumption of equidistance is not essential, but simplifies the presentation of the method and the proofs. There is no loss of generality to assume the

${ }^{*}$ Corresponding author Abdelkader Mokkadem : abdelkader.mokkadem@uvsq.fr

Mariane Pelletier : mariane.pelletier@uvsq.fr 
random variables $\left(\varepsilon_{i, n}\right)_{1 \leq i \leq n, n \geq 1}$ are defined in the same probability space, e.g. in the product space $\prod_{n \geq 1} \mathbb{R}^{n}$ endowed with the probability $\mathbb{P}=\otimes_{n \geq 1} \mathbb{P}_{\varepsilon}^{\otimes n}$ where $\mathbb{P}_{\varepsilon}$ is the probability law of $\varepsilon_{i, n}$.

For sake of simplicity, we omit the index $n$, and write:

$$
Y_{i}=\mu\left(t_{i}\right)+\varepsilon_{i}, i=1, \ldots, n .
$$

A large amount of literature is devoted to the study of the model (1), in particular to construct hypothesis tests and confidence bands based on asymptotic convergence to the normal distribution. Among many others, let us cite Davison and Tsai (1992), Dette and Munk (1998a), Dette and Munk (1998b), Dette et al. (1998), Dieboldt (1995), Eubank and Hart (1992), Eubank and Spiegelmann (1990), Gasser et al. (1986), Härdle and Mammen (1993), Härdle and Marron (1990), Mohdeb and Mokkadem (2004), Mohdeb and Mokkadem (2015), Munk and Dette (1998) and Stute (1997). The use of moderate deviations principles (MDP) for the construction of confidence bands has been introduced by Mokkadem and Pelletier (2006) in the random design set up. Our purpose is to extend this approach to the case of fixed design model (1). Based on the sample $(t i, Y i), i=1, \ldots, n$, we define the kernel estimator $\mu_{n}$ of $\mu$ by setting

$$
\mu_{n}(t)=\frac{1}{n h_{n}} \sum_{i=1}^{n} Y_{i} K\left(\frac{t-t_{i}}{h_{n}}\right)
$$

where the bandwidth $\left(h_{n}\right)$ is a sequence of positive real numbers that goes to zero, and where the kernel $K$ is a function satisfying $\int_{\mathbb{R}} K(z) d z=1$.

Let us recall the concept of large and moderate deviations. A function $J: \mathbb{R} \rightarrow[0, \infty]$ is called a good rate function if, for each $M<\infty$, the level set $\{x \in \mathbb{R}: J(x) \leq M\}$ is a compact set. A $\mathbb{R}$-valued sequence $\left(Z_{n}\right)_{n \geq 1}$ satisfies a large deviations principle (LDP) with speed $\left(\nu_{n}\right)$ and good rate function $J$ if:

(a) $\left(\nu_{n}\right)$ is a positive sequence such that $\lim _{n \rightarrow \infty} \nu_{n}=\infty$,

(b) for every Borel set $B \subset \mathbb{R}$,

$$
\begin{aligned}
-\inf _{x \in \breve{B}} J(x) & \leq \liminf _{n \rightarrow \infty} \nu_{n}^{-1} \log \mathbb{P}\left(Z_{n} \in B\right) \\
& \leq \limsup _{n \rightarrow \infty} \nu_{n}^{-1} \log \mathbb{P}\left(Z_{n} \in B\right) \leq-\inf _{x \in \bar{B}} J(x),
\end{aligned}
$$

where $\breve{B}$ and $\bar{B}$ denote the interior and the closure of $B$ respectively. Let $\left(v_{n}\right)$ be a nonrandom sequence that goes to infinity. If the sequence $\left(v_{n} Z_{n}\right)_{n \geq 1}$ satisfies a LDP, we say that $\left(Z_{n}\right)_{n \geq 1}$ satisfies a MDP.

The main purpose of this paper is to establish pointwise and uniform MDP for the sequence $\left(\mu_{n}-\mu\right)$. For any function $G$ and positive sequence $\left(u_{n}\right)$, set

$$
I\left(G, t, u_{n}\right)=\frac{1}{n u_{n}} \sum_{i=1}^{n} G^{2}\left(\frac{t-t_{i}}{u_{n}}\right),
$$

and let $\left(v_{n}\right)$ be a nonrandom sequence that goes to infinity. For $\left.t \in\right] 0,1[$, we give conditions under which the sequence

$$
\left(v_{n} \frac{\left(\mu_{n}(t)-\mu(t)\right)}{\sqrt{I\left(K^{2}, t, h_{n}\right)}}\right)
$$

Journal home page: www.jafristat.net ; www.projecteuclid.org/as 
satisfies a LDP with speed $\left(n h_{n} / v_{n}^{2}\right)$ and good rate function

$$
J: x \mapsto \frac{x^{2}}{2 \sigma^{2}} .
$$

Moreover, let $\left(\rho_{n}\right)$ be a nonrandom positive sequence that goes to zero. We give conditions under which the sequence

$$
\left(v_{n} \sup _{t \in\left[\rho_{n}, 1-\rho_{n}\right]} \frac{\left|\mu_{n}(t)-\mu(t)\right|}{\sqrt{I\left(K^{2}, t, h_{n}\right)}}\right)
$$

satisfies a LDP with speed $\left(n h_{n} / v_{n}^{2}\right)$ and good rate function $J$ on $\mathbb{R}^{+}$.

Large and moderate deviations principles have been widely applied in statistical inference. Their use has been initiated by the papers of Chernov (1952) and Bahadur (1960), and then developped in various directions. Let us cite, among many others, Borovkov and Mogulskii (1992), Groeneboom (1980), Ibragimov and Radavicius (1981), Kallengerg (1982), Kallengerg (1983a), Kallengerg (1983b), Korostelev and Leonov (1995), Nikitin (1995), Mokkadem and Pelletier (2006), and Puhalskii and Spokoiny (1998). In Section 2.2, we provide an application of our MDP results to the construction of confidence regions for $\mu$.

Our paper is now organized as follows. Our assumptions and main results are stated in Section 2. Section 3 is deserved to the proofs.

\section{Assumptions and Main Results}

The assumptions to which we will refer in the sequel are the following.

(A1) i) $K$ is a nonnegative and Lipschitz function on $\mathbb{R}$ such that

$$
\int_{\mathbb{R}} K(z) d z=1, \quad \int_{\mathbb{R}} z K(z) d z=0, \quad \text { and } \quad \int_{\mathbb{R}} z^{2} K(z) d z<\infty .
$$

ii) There exists $m>3$ such that $\lim _{|z| \rightarrow \infty}|z|^{m} K(z)=0$.

(A2) i) $\mu$ is Lipschitz on $[0,1]$.

ii) $\mu$ is twice differentiable on $] 0,1\left[\sup _{t \in] 0,1[}\left|\mu^{\prime}(t)\right|<\infty\right.$, and $\sup _{t \in] 0,1[}\left|\mu^{\prime \prime}(t)\right|<\infty$.

(A3) There exists $a>0$ such that $\mathbb{E}\left(\exp \left(a\left|\varepsilon_{i}\right|\right)\right)<\infty$.

\subsection{Moderate deviations principles}

Our main results are the two following theorems.

Theorem 1 (Pointwise MDP). Let $(A 1),(A 2) i)$, and $(A 3)$ hold, and set $t \in] 0,1[$. For all sequences $\left(h_{n}\right)$ and $\left(v_{n}\right)$ satisfying the conditions

$$
v_{n} \rightarrow \infty, \quad \frac{n h_{n}}{v_{n}^{2}} \rightarrow \infty, \quad \text { and } \quad \frac{v_{n}}{n h_{n}^{2}} \rightarrow 0,
$$


the sequence

$$
\left(v_{n} \frac{\left(\mu_{n}(t)-\mathbb{E}\left(\mu_{n}(t)\right)\right)}{\sqrt{I\left(K^{2}, t, h_{n}\right)}}\right)
$$

satisfies a LDP with speed $\left(n h_{n} / v_{n}^{2}\right)$ and good rate function $J$ on $\mathbb{R}$.

Theorem 2 (Uniform MDP). Let $(A 1),(A 2) i)$, and $(A 3)$ hold, assume that $\left(h_{n}\right)$ and $\left(v_{n}\right)$ satisfy the conditions

$$
v_{n} \rightarrow \infty, \quad \frac{n h_{n}}{v_{n}^{2} \log \left(1 / h_{n}\right)} \rightarrow \infty, \quad \text { and } \quad \frac{v_{n}}{n h_{n}^{2}} \rightarrow 0,
$$

and let $\left(\rho_{n}\right)$ be such that

$$
0<\rho_{n}<\frac{1}{2}, \quad \rho_{n} \rightarrow 0, \quad \frac{\rho_{n}}{h_{n}} \rightarrow \infty, \quad \text { and } \quad v_{n}\left(\frac{h_{n}}{\rho_{n}}\right)^{m-2} \rightarrow 0 .
$$

Then, the sequence

$$
\left(v_{n} \sup _{t \in\left[\rho_{n}, 1-\rho_{n}\right]} \frac{\left|\mu_{n}(t)-\mathbb{E}\left(\mu_{n}(t)\right)\right|}{\sqrt{I\left(K^{2}, t, h_{n}\right)}}\right)
$$

satisfies a LDP with speed $\left(n h_{n} / v_{n}^{2}\right)$ and good rate function $J$ on $\mathbb{R}^{+}$.

Let us point out that, to obtain the uniform MDP, we require the condition $n h_{n}\left[v_{n}^{2} \log \left(1 / h_{n}\right)\right]^{-1} \rightarrow \infty$ in $(3)$, whereas, for the pointwise MDP, the slightly weaker condition $n h_{n} v_{n}^{-2} \rightarrow \infty$ in (2) is sufficient. This difference of assumptions between the pointwise case and the uniform case is usual in the nonparametric framework. It is of course completely explained by the difference between the pointwise and the uniform weak asymptotic behaviours of the kernel estimator $\mu_{n}$. As a matter of fact, let us recall that, for $\left.t \in\right] 0,1[$, $\mu_{n}(t)$ is known to satisfy the central limit theorem (CLT)

$$
Z_{n}(t)=\frac{\sqrt{n h_{n}}\left(\mu_{n}(t)-\mathbb{E}\left(\mu_{n}(t)\right)\right)}{\sqrt{I\left(K^{2}, t, h_{n}\right)}} \stackrel{\mathcal{D}}{\rightarrow} \mathcal{N}\left(0, \sigma^{2}\right),
$$

(where $\stackrel{\mathcal{D}}{\rightarrow}$ denotes the convergence in distribution) and that, for $\rho \in] 0,1 / 2[$, Stadtmüller (1986) proved that

$$
\sqrt{2 \log \left(1 / h_{n}\right)}\left[\sup _{t \in[\rho, 1-\rho]}\left|Z_{n}(t)\right|-\sqrt{2 \log \left(1 / h_{n}\right)}\left(1+\eta_{n}\right)\right] \stackrel{\mathcal{D}}{\rightarrow} Z^{*}
$$

where $Z^{*}$ is a random variable whose distribution function is $z \mapsto \exp (-2 \exp (-z))$, and where $\left(\eta_{n}\right)$ is a nonrandom sequence (explicitly given in Stadtmüller (1986)) satisfying $\lim _{n \rightarrow \infty} \eta_{n}=0$. It follows that, for the sequences $\left(v_{n}\right)$ such that $n h_{n} v_{n}^{-2} \rightarrow \infty$ and $n h_{n}\left[v_{n}^{2} \log \left(1 / h_{n}\right)\right]^{-1} \rightarrow 0$, the sequence $\left(v_{n}\left[\mu_{n}(t)-\mathbb{E}\left(\mu_{n}(t)\right)\right]\right)$ converges in probability to zero, whereas the sequence $\left(v_{n} \sup _{t \in\left[\rho_{n}, 1-\rho_{n}\right]}\left|\mu_{n}(t)-\mathbb{E}\left(\mu_{n}(t)\right)\right|\right)$ does not, so that a

Journal home page: www.jafristat.net; www.projecteuclid.org/as 
pointwise MDP for $\mu_{n}$ exists, but not a uniform MDP.

It is often more convenient to have MDP for $\mu_{n}-\mu$ rather than for $\mu_{n}-\mathbb{E}\left(\mu_{n}\right)$ : this is the object of the following corollaries.

Corollary 1 (Pointwise MDP). Let $(A 1),(A 2) i)$, and $(A 3)$ hold. Set $t \in] 0,1[$, and assume that $\mu$ is twice differentiable at $t$. Then, for all sequences $\left(h_{n}\right)$ and $\left(v_{n}\right)$ satisfying the conditions in (2) and such that $v_{n} h_{n}^{2} \rightarrow 0$, the sequence

$$
\left(v_{n} \frac{\left(\mu_{n}(t)-\mu(t)\right)}{\sqrt{I\left(K^{2}, t, h_{n}\right)}}\right)
$$

satisfies a LDP with speed $\left(n h_{n} / v_{n}^{2}\right)$ and good rate function $J$ on $\mathbb{R}$.

Corollary 2 (Uniform MDP). Let $(A 1)-(A 3)$ hold. Then, for all sequences $\left(h_{n}\right)$ and $\left(v_{n}\right)$ satisfying the conditions in (3) and such that $v_{n} h_{n}^{2} \rightarrow 0$, and for all sequences $\left(\rho_{n}\right)$ satisfying the conditions in (4), the sequence

$$
\left(v_{n} \sup _{t \in\left[\rho_{n}, 1-\rho_{n}\right]} \frac{\left|\mu_{n}(t)-\mu(t)\right|}{\sqrt{I\left(K^{2}, t, h_{n}\right)}}\right)
$$

satisfies a LDP with speed $\left(n h_{n} / v_{n}^{2}\right)$ and good rate function $J$ on $\mathbb{R}^{+}$.

\subsection{Application to the construction of confidence regions}

Moderate deviations principles allow to construct confidence regions with positive logarithmic asymptotic level. These confidence regions have an asymptotic level equal to one. Moreover, they often are asymptotic almost sure sequences of confidence regions. Let us recall the notion of the logarithmic asymptotic level and of asymptotic almost sure sequences of confidence regions, as defined in Mokkadem and Pelletier (2006).

Let $\left\{D_{n}\right\}$ be a sequence of confidence regions of an unknown parameter $\theta$, and let $\Omega$ denote the underlying probability space. The logarithmic asymptotic level of $\left\{D_{n}\right\}$ is $\gamma(\gamma>0)$ with speed $w_{n}\left(w_{n} \rightarrow \infty\right)$ if

$$
\lim _{n \rightarrow \infty} \frac{1}{w_{n}} \log \mathbb{P}\left(\theta \notin D_{n}\right)=-\gamma .
$$

Moreover, $\left\{D_{n}\right\}$ is an asymptotic almost sure (or consistent) sequence of confidence regions of $\theta$ if there exists $\Omega_{0} \subset \Omega$ such that:

$$
\left\{\begin{array}{l}
\bullet \mathbb{P}\left(\Omega_{0}\right)=1 \\
\bullet \forall \omega \in \Omega_{0}, \exists N(\omega) \text { such that } n \geq N(\omega) \Rightarrow \theta \in D_{n}(\omega) .
\end{array}\right.
$$

To deduce confidence regions for $\mu$ from Corollaries 1 and 2 , we need to estimate the variance $\sigma^{2}$ of the errors $\varepsilon_{i}$ : we use the estimator

$$
\hat{\sigma}_{n}^{2}=\frac{1}{n-2} \sum_{i=2}^{n-1} \frac{2}{3}\left(\frac{1}{2} Y_{i-1}+\frac{1}{2} Y_{i+1}-Y_{i}\right)^{2}
$$

Journal home page: www.jafristat.net ; www.projecteuclid.org/as 
introduced by Gasser et al. (1986). Moreover, we need to strenghten Assumption (A3) in the following way.

$\left(A^{\prime} 3\right)$ There exists $a>0$ such that $\mathbb{E}\left(\exp \left(a \varepsilon_{i}^{2}\right)\right)<\infty$.

\subsubsection{Application to the construction of confidence intervals}

The pointwise MDP given in Corollary 1 allows to prove the following proposition.

Proposition 1. Let $(A 1),(A 2) i)$ and $\left(A^{\prime} 3\right)$ hold. Set $\left.t \in\right] 0,1[$, assume that $\mu$ is twice differentiable at $t$, and let $\left(h_{n}\right)$ and $\left(v_{n}\right)$ satisfy the conditions in (2) and be such that $v_{n} h_{n}^{2} \rightarrow 0$. Then, for any $\delta>0$, we have

$\lim _{n \rightarrow \infty} \frac{v_{n}^{2}}{n h_{n}} \log \mathbb{P}\left(\mu(t) \notin\left[\mu_{n}(t)-\delta \frac{\hat{\sigma}_{n} \sqrt{I\left(K^{2}, t, h_{n}\right)}}{v_{n}}, \mu_{n}(t)+\delta \frac{\hat{\sigma}_{n} \sqrt{I\left(K^{2}, t, h_{n}\right)}}{v_{n}}\right]\right)=-\frac{\delta^{2}}{2}$.

Moreover, if the additional condition $n h_{n}\left[v_{n}^{2} \log \left(1 / h_{n}\right)\right]^{-1} \rightarrow \infty$ holds, then the sequence of intervals

$$
\left[\mu_{n}(t)-\delta \frac{\hat{\sigma}_{n} \sqrt{I\left(K^{2}, t, h_{n}\right)}}{v_{n}}, \mu_{n}(t)+\delta \frac{\hat{\sigma}_{n} \sqrt{I\left(K^{2}, t, h_{n}\right)}}{v_{n}}\right]
$$

is an asymptotic almost sure sequence of confidence intervals of $\mu(t)$.

Remark 1. Proposition 1 holds in particular by choosing $\left(h_{n}\right)=\left(n^{-a}\right), 0<a<1 / 2$, and $\left(v_{n}\right)=\left(n^{b}\right)$ with $0<b<\min \{2 a, 1-2 a,(1-a) / 2\}$.

Before comparing the confidence intervals given by Proposition 1 with those obtained by application of the CLT, let us first state the following corollary, which says that the quantity $I\left(K^{2}, t, h_{n}\right)$ in the bounds of the confidence intervals of Proposition 1 can be replaced by other quantities.

Corollary 3. Let $\left(h_{n}^{*}\right)$ be a bandwidth such that $\lim _{n \rightarrow \infty} h_{n}^{*}=0$ and $\lim _{n \rightarrow \infty} n h_{n}^{* 2}=\infty$. Throughout Proposition 1, the quantity $I\left(K^{2}, t, h_{n}\right)$ can be replaced either by $I\left(K^{2}, t, h_{n}^{*}\right)$ or by $\int_{\mathbb{R}} K^{2}(s) d s$.

Hall (1992) shows that, to construct confidence intervals, slightly undersmoothing is more efficient than bias estimation. Let $\left(h_{n}^{*}\right)$ be a bandwidth and $\mu_{n}^{*}$ be the kernel estimator of $\mu$ computed with this bandwidth $\left(\mu_{n}^{*}(t)=\left(n h_{n}^{*}\right)^{-1} \sum_{i=1}^{n} Y_{i} K\left[\left(h_{n}^{*}\right)^{-1}\left(t-t_{i}\right)\right]\right)$. To construct confidence intervals for $\mu$ by applying the CLT and by slightly undersmoothing, $\left(h_{n}^{*}\right)$ must be chosen such that $n h_{n}^{* 5} \rightarrow 0$ (and, for instance, such that $n h_{n}^{* 3} \rightarrow \infty$ ), in which case $\mu_{n}^{*}(t)$ is known to satisfy the CLT

$$
\frac{\sqrt{n h_{n}^{*}}\left(\mu_{n}^{*}(t)-\mu(t)\right)}{\sigma \sqrt{I\left(K^{2}, t, h_{n}^{*}\right)}} \stackrel{\mathcal{D}}{\rightarrow} \mathcal{N}(0,1) .
$$

Journal home page: www.jafristat.net; www.projecteuclid.org/as 
Set $\alpha \in] 0,1\left[\right.$, and let $t_{\alpha / 2}$ be the $\alpha / 2$-quantile of the standard normal distribution. The estimator $\hat{\sigma}_{n}^{2}$ of Gasser et al. (1986) being consistent, it follows that

$$
\mathcal{I}_{n, \alpha}^{*}(t)=\left[\mu_{n}^{*}(t)-t_{\alpha / 2} \frac{\hat{\sigma}_{n} \sqrt{I\left(K^{2}, t, h_{n}^{*}\right)}}{\sqrt{n h_{n}^{*}}}, \mu_{n}^{*}(t)+t_{\alpha / 2} \frac{\hat{\sigma}_{n} \sqrt{I\left(K^{2}, t, h_{n}^{*}\right)}}{\sqrt{n h_{n}^{*}}}\right]
$$

is a confidence interval for $\mu(t)$ with asymptotic level $1-\alpha$. Now, let $\left(u_{n}\right)$ be a positive sequence such that $u_{n} \rightarrow \infty$ and $n h_{n}^{* 5} u_{n}^{4} \rightarrow 0$, set $\left(h_{n}\right)=\left(u_{n} h_{n}^{*}\right)$ in the definition of $\mu_{n}(t)$ and

$$
\mathcal{I}_{n}(t)=\left[\mu_{n}(t)-t_{\alpha / 2} \frac{\hat{\sigma}_{n} \sqrt{I\left(K^{2}, t, h_{n}^{*}\right)}}{\sqrt{n h_{n}^{*}}}, \mu_{n}(t)+t_{\alpha / 2} \frac{\hat{\sigma}_{n} \sqrt{I\left(K^{2}, t, h_{n}^{*}\right)}}{\sqrt{n h_{n}^{*}}}\right] .
$$

The interval $\mathcal{I}_{n}(t)$ is simply the translation of $\mathcal{I}_{n, \alpha}^{*}(t)$ from the (random) quantity $\mu_{n}(t)-$ $\mu_{n}^{*}(t)$ : it has the same width as the interval $\mathcal{I}_{n, \alpha}^{*}(t)$, but its asymptotic level clearly equals one (and not $1-\alpha<1$ ). The application of Corollary 3 ensures that its logarithmic asymptotic level equals $t_{\alpha / 2}^{2} / 2$ with speed $u_{n}$. Let us point out that this implies that, for $\eta>0$ and $n$ large enough,

$$
\exp \left(-\left[\frac{t_{\alpha / 2}^{2}}{2}+\eta\right] u_{n}\right) \leq \mathbb{P}\left(\mu(t) \notin \mathcal{I}_{n}(t)\right) \leq \exp \left(-\left[\frac{t_{\alpha / 2}^{2}}{2}-\eta\right] u_{n}\right) .
$$

The convergence rate of the level of $\mathcal{I}_{n}(t)$ toward one is thus larger when $\left(u_{n}\right)$ goes faster to infinity. Of course, $\left(u_{n}\right)$ cannot be chosen arbitrarily large because of the condition $n h_{n}^{* 5} u_{n}^{4} \rightarrow$ 0 . However, it is interesting to note that it is always possible (and advised) to choose $\left(u_{n}\right)$ large enough so that $n h_{n}^{5} \rightarrow \infty$. In this case, the interval $\mathcal{I}_{n}(t)$ is centered on an estimator $\mu_{n}(t)$ for which there is no CLT (the sequence $\left(h_{n}^{-2}\left[\mu_{n}(t)-\mu(t)\right]\right)$ then converging to a usually nonzero constant), but the convergence rate of its level toward one is larger than when $\mathcal{I}_{n}(t)$ is centered on estimators for which a CLT exists.

\subsubsection{Application to the construction of confidence bands}

The uniform MDP given in Corollary 2 allows to prove the following proposition.

Proposition 2. Let $(A 1),(A 2)$ and $\left(A^{\prime} 3\right)$ hold, assume that $\left(h_{n}\right),\left(v_{n}\right)$, and $\left(\rho_{n}\right)$ satisfy the conditions in (3) and (4), and that $v_{n} h_{n}^{2} \rightarrow 0$. Then, for any $\delta>0$, we have

$$
\begin{aligned}
\lim _{n \rightarrow \infty} \frac{v_{n}^{2}}{n h_{n}} \log \mathbb{P}\left(\exists t \in\left[\rho_{n}, 1-\rho_{n}\right], \mu(t) \notin\left[\mu_{n}(t)-\delta \frac{\hat{\sigma}_{n} \sqrt{I\left(K^{2}, t, h_{n}\right)}}{v_{n}},\right.\right. \\
\left.\left.\mu_{n}(t)+\delta \frac{\hat{\sigma}_{n} \sqrt{I\left(K^{2}, t, h_{n}\right)}}{v_{n}}\right]\right)=-\frac{\delta^{2}}{2} .
\end{aligned}
$$

Moreover, the sequence of sets of functions

$$
D_{n}=\left\{g:[0,1] \rightarrow \mathbb{R},\left|g(t)-\mu_{n}(t)\right| \leq \delta \frac{\hat{\sigma}_{n} \sqrt{I\left(K^{2}, t, h_{n}\right)}}{v_{n}} \forall t \in\left[\rho_{n}, 1-\rho_{n}\right]\right\}
$$

is an asymptotic almost sure sequence of confidence regions of $\mu$.

Journal home page: www.jafristat.net ; www.projecteuclid.org/as 
Remark 2. Proposition 2 holds in particular by choosing a Gaussian kernel $K$ or a kernel $K$ with bounded support, $\left(h_{n}\right)=\left(n^{-a}\right)$ with $0<a<1 / 2,\left(v_{n}\right)=\left(n^{b}\right)$ with $0<b<$ $\min \{2 a, 1-2 a,(1-a) / 2\}$, and $\left(\rho_{n}\right)=\left(n^{r}\right)$ with $0<r<a$.

The quantity $I\left(K^{2}, t, h_{n}\right)$ appearing in the bounds of confidence bands of Proposition 2 can be replaced by other quantities, as stated in the following corollary.

Corollary 4. Let $\left(h_{n}^{*}\right)$ be a bandwidth such that $\lim _{n \rightarrow \infty} n h_{n}^{* 2}=\infty$ and $\lim _{n \rightarrow \infty} \rho_{n} / h_{n}^{*}=$ $\infty$. Throughout Proposition 2, the quantity $I\left(K^{2}, t, h_{n}\right)$ can be replaced either by $I\left(K^{2}, t, h_{n}^{*}\right)$ or by $\int_{\mathbb{R}} K^{2}(s) d s$.

Let us note that the confidence bands with logarithmic asymptotic level $\delta^{2} / 2$ with speed $\left(n h_{n} / v_{n}^{2}\right)$ provided by Proposition 2 (or by Corollary 4) are unions of confidence intervals, which all share the same logarithmic asymptotic level that also equals $\delta^{2} / 2$ with speed $\left(n h_{n} / v_{n}^{2}\right)$.

To conclude this section, we now compare the confidence bands obtained by application of the MDP with those constructed with the help of result (5) of Stadtmüller (1986).

Let $\left(h_{n}^{*}\right)$ be a bandwidth, and let $\mu_{n}^{*}$ be the kernel estimator of $\mu$ computed with the help of this bandwidth. To construct confidence bands by applying the result (5) of Stadtmüller (1986) and by slightly undersmoothing, $\left(h_{n}^{*}\right)$ must be chosen such that $n h_{n}^{* 5} \log \left(1 / h_{n}^{*}\right) \rightarrow 0$ (and, let us say, such that $\left.n h_{n}^{* 3} \log \left(1 / h_{n}^{*}\right) \rightarrow \infty\right)$. Set $\left.\rho \in\right] 0,1 / 2\left[, z_{\alpha}\right.$ such that $\exp \left(-2 \exp \left(-z_{\alpha}\right)\right)=1-\alpha$ and, for all $t \in[\rho, 1-\rho]$,

$$
\begin{aligned}
\mathcal{I}_{n}^{*}(t)= & {\left[\mu_{n}^{*}(t)-\frac{\hat{\sigma}_{n} \sqrt{I\left(K^{2}, t, h_{n}^{*}\right)}}{\sqrt{n h_{n}^{*}}} \sqrt{\log \left(1 / h_{n}^{*}\right)}\left(\sqrt{2}+\eta_{n}+\frac{z_{\alpha}}{\sqrt{2} \log \left(1 / h_{n}^{*}\right)}\right),\right.} \\
& \left.\mu_{n}^{*}(t)+\frac{\hat{\sigma}_{n} \sqrt{I\left(K^{2}, t, h_{n}^{*}\right)}}{\sqrt{n h_{n}^{*}}} \sqrt{\log \left(1 / h_{n}^{*}\right)}\left(\sqrt{2}+\eta_{n}+\frac{z_{\alpha}}{\sqrt{2} \log \left(1 / h_{n}^{*}\right)}\right)\right] .
\end{aligned}
$$

The union (on $t \in[\rho, 1-\rho]$ ) of the confidence intervals $\mathcal{I}_{n}^{*}(t)$ is a confidence band for $\mu$ with asymptotic level $1-\alpha$, since the result (5) of Stadtmüller (1986) guarantees that

$$
\lim _{n \rightarrow \infty} \mathbb{P}\left(\forall t \in[\rho, 1-\rho], \mu(t) \in \mathcal{I}_{n}^{*}(t)\right)=1-\alpha .
$$

Let us point out that the asymptotic level of each confidence interval $\mathcal{I}_{n}^{*}(t)$ is clearly 1 . More precisely, the application of Corollary $3\left(\right.$ with $\left(h_{n}\right)=\left(h_{n}^{*}\right)$ and $\left(v_{n}\right)=\left(\sqrt{n h_{n}^{*} / \log \left(1 / h_{n}^{*}\right)}\right)$, together with a continuity argument, ensures that, for all $t \in[\rho, 1-\rho]$, the logarithmic asymptotic level of $\mathcal{I}_{n}^{*}(t)$ equals 1 with speed $\log \left(1 / h_{n}^{*}\right)$. There is thus a big difference between the confidence bands obtained by application of the MDP and those constructed with the help of the asymptotic law of the normalized uniform errors: when MDP are used, the confidence intervals whose union gives the confidence band, and the confidence band itself, share the same (logarithmic) asymptotic level, contrary to the case the result (5) is used.

Journal home page: www.jafristat.net ; www.projecteuclid.org/as 
Now, let $\left(u_{n}\right)$ be a positive sequence such that $u_{n} \rightarrow \infty$ and $n h_{n}^{* 5}\left[\log \left(1 / h_{n}^{*}\right)\right]^{-1} u_{n}^{4} \rightarrow 0$, set $\left(h_{n}\right)=\left(u_{n} h_{n}^{*}\right)$ and, for $t \in[\rho, 1-\rho]$,

$$
\begin{aligned}
\mathcal{I}_{n}(t)= & {\left[\mu_{n}(t)-\frac{\hat{\sigma}_{n} \sqrt{I\left(K^{2}, t, h_{n}^{*}\right)}}{\sqrt{n h_{n}^{*}}} \sqrt{\log \left(1 / h_{n}^{*}\right)}\left(\sqrt{2}+\eta_{n}+\frac{z_{\alpha}}{\sqrt{2} \log \left(1 / h_{n}^{*}\right)}\right),\right.} \\
& \left.\mu_{n}(t)+\frac{\hat{\sigma}_{n} \sqrt{I\left(K^{2}, t, h_{n}^{*}\right)}}{\sqrt{n h_{n}^{*}}} \sqrt{\log \left(1 / h_{n}^{*}\right)}\left(\sqrt{2}+\eta_{n}+\frac{z_{\alpha}}{\sqrt{2} \log \left(1 / h_{n}^{*}\right)}\right)\right] .
\end{aligned}
$$

For each $t \in[\rho, 1-\rho], \mathcal{I}_{n}(t)$ is the translation of $\mathcal{I}_{n}^{*}(t)$ from the quantity $\mu_{n}(t)-\mu_{n}^{*}(t)$. The application of Corollaries 3 and 4, together with a continuity argument, ensures that the confidence intervals $\mathcal{I}_{n}(t)$ and the confidence band defined as the union (for $t \in[\rho, 1-\rho]$ ) of the intervals $\mathcal{I}_{n}(t)$ have a logarithmic asymptotic level equal to 1 with speed $u_{n} \log \left(1 / h_{n}^{*}\right)$. In particular, this implies that, for $\eta>0$ and $n$ large enough,

$\exp \left(-[1+\eta] u_{n} \log \left(1 / h_{n}^{*}\right)\right) \leq \mathbb{P}\left(\exists t \in[\rho, 1-\rho], \mu(t) \notin \mathcal{I}_{n}(t)\right) \leq \exp \left(-[1-\eta] u_{n} \log \left(1 / h_{n}^{*}\right)\right)$.

The application of MDP thus allows to get confidence bands, which have the same width as those constructed by using the result (5) of Stadtmüller (1986), but whose asymptotic level is better. Let us point out that it is preferable again to choose $\left(u_{n}\right)$ going to infinity as fast as possible (but such that $n h_{n}^{* 5}\left[\log \left(1 / h_{n}^{*}\right)\right]^{-1} u_{n}^{4} \rightarrow 0$ ), and thus to center the intervals $\mathcal{I}_{n}$ on an estimator $\mu_{n}$ for which the result (5) does not hold. Let us finally note that the simplified intervals

$\mathcal{I}_{n}^{\prime}(t)=\left[\mu_{n}(t)-\frac{\hat{\sigma}_{n} \sqrt{I\left(K^{2}, t, h_{n}^{*}\right)}}{\sqrt{n h_{n}^{*}}} \sqrt{2 \log \left(1 / h_{n}^{*}\right)}, \mu_{n}(t)+\frac{\hat{\sigma}_{n} \sqrt{I\left(K^{2}, t, h_{n}^{*}\right)}}{\sqrt{n h_{n}^{*}}} \sqrt{2 \log \left(1 / h_{n}^{*}\right)}\right]$

and the confidence band defined as the union (for $t \in[\rho, 1-\rho]$ ) of the intervals $\mathcal{I}_{n}^{\prime}(t)$ have the same logarithmic asymptotic level as the intervals $\mathcal{I}_{n}(t)$ (and the associated confidence band).

\section{Proofs}

Throughout the proofs, we use the following notation. For any function $G$, we set

$$
\begin{aligned}
I(G) & =\int_{\mathbb{R}} G(s) d s, \\
\|G\|_{\infty} & =\sup _{t \in \mathbb{R}}|G(t)| .
\end{aligned}
$$

Moreover, if $G$ is Lipschitz, we denote by $\|G\|_{L}$ its Lipschitz norm.

\subsection{Proof of Theorems 1 and 2}

In order to prove Theorems 1 and 2, we need the following preliminary technical lemmas.

Lemma 1. Let $G$ be an integrable Lipschitz function, and let $\left(h_{n}\right)$ and $\left(\rho_{n}\right)$ satisfy the conditions

$$
\lim _{n \rightarrow \infty} n h_{n}^{2}=\infty, \quad \lim _{n \rightarrow \infty} \rho_{n}=0, \quad \text { and } \quad \lim _{n \rightarrow \infty} \frac{\rho_{n}}{h_{n}}=\infty .
$$

Journal home page: www.jafristat.net ; www.projecteuclid.org/as 
Then, we have

$$
\lim _{n \rightarrow \infty} \sup _{t \in\left[\rho_{n}, 1-\rho_{n}\right]}\left|I\left(G, t, h_{n}\right)-I(G)\right|=0 .
$$

Lemma 2. Set

$$
\Lambda_{n, t}(u)=\frac{v_{n}^{2}}{n h_{n}} \log \mathbb{E}\left[\exp \left(\frac{n h_{n} u\left(\mu_{n}(t)-\mu(t)\right)}{v_{n} \sqrt{I\left(K^{2}, t, h_{n}\right)}}\right)\right]
$$

1) Under the assumptions of Theorem 1, we have

$$
\lim _{n \rightarrow \infty} \Lambda_{n, t}(u)=\frac{u^{2} \sigma^{2}}{2} .
$$

2) Under the assumptions of Theorem 2, we have

$$
\lim _{n \rightarrow \infty} \sup _{t \in\left[\rho_{n}, 1-\rho_{n}\right]}\left|\Lambda_{n, t}(u)-\frac{u^{2} \sigma^{2}}{2}\right|=0 .
$$

Lemmas 1 and 2 are established in Sections 3.1.1 and 3.1.2, respectively. Then, Theorems 1 and 2 are proved in Sections 3.1.3 and 3.1.4, respectively.

\subsubsection{Proof of Lemma 1}

Since $t_{i}=(i-1) / n, i \in\{1, \ldots, n\}$, we have, by setting $t_{n+1}=1$,

$$
\begin{aligned}
\left|I\left(G, t, h_{n}\right)-\frac{1}{h_{n}} \int_{0}^{1} G\left(\frac{t-s}{h_{n}}\right) d s\right| & =\frac{1}{h_{n}}\left|\sum_{i=1}^{n} \int_{t_{i}}^{t_{i+1}}\left[G\left(\frac{t-t_{i}}{h_{n}}\right)-G\left(\frac{t-s}{h_{n}}\right)\right] d s\right| \\
& \leq \frac{\|G\|_{L}}{h_{n}^{2}} \sum_{i=1}^{n} \int_{t_{i}}^{t_{i+1}}\left(s-t_{i}\right) d s \\
& \leq \frac{\|G\|_{L}}{2 h_{n}^{2}} \sum_{i=1}^{n}\left(t_{i+1}-t_{i}\right)^{2} \\
& \leq \frac{\|G\|_{L}}{2 n h_{n}^{2}}
\end{aligned}
$$

Moreover, we note that

$$
\begin{aligned}
\left|\frac{1}{h_{n}} \int_{0}^{1} G\left(\frac{t-s}{h_{n}}\right) d s-\int_{\mathbb{R}} G(u) d u\right| & =\left|\int_{-(1-t) / h_{n}}^{t / h_{n}} G(u) d u-\int_{\mathbb{R}} G(u) d u\right| \\
& \leq\left|\int_{t / h_{n}}^{\infty} G(u) d u\right|+\left|\int_{-\infty}^{-(1-t) / h_{n}} G(u) d u\right|,
\end{aligned}
$$

so that

$$
\begin{aligned}
\sup _{t \in\left[\rho_{n}, 1-\rho_{n}\right]}\left|\frac{1}{h_{n}} \int_{0}^{1} G\left(\frac{t-s}{h_{n}}\right) d s-\int_{\mathbb{R}} G(u) d u\right| & \leq \int_{\rho_{n} / h_{n}}^{\infty}|G(u)| d u+\int_{-\infty}^{-\rho_{n} / h_{n}}|G(u)| d u \\
& \rightarrow 0 \text { as } n \rightarrow \infty .
\end{aligned}
$$

Journal home page: www.jafristat.net ; www.projecteuclid.org/as 
It follows that

$$
\begin{aligned}
\sup _{t \in\left[\rho_{n}, 1-\rho_{n}\right]}\left|I\left(G, t, h_{n}\right)-I(G)\right| \leq & \sup _{t \in\left[\rho_{n}, 1-\rho_{n}\right]}\left|I\left(G, t, h_{n}\right)-\frac{1}{h_{n}} \int_{0}^{1} G\left(\frac{t-s}{h_{n}}\right) d s\right| \\
& +\sup _{t \in\left[\rho_{n}, 1-\rho_{n}\right]}\left|\frac{1}{h_{n}} \int_{0}^{1} G\left(\frac{t-s}{h_{n}}\right) d s-\int_{\mathbb{R}} G(u) d u\right| \\
& \rightarrow 0 \text { as } n \rightarrow \infty,
\end{aligned}
$$

which gives Lemma 1.

\subsubsection{Proof of Lemma 2}

In order to give a unified proof for both parts of Lemma 2, we set $\mathcal{E}_{n}= \begin{cases}\{t\} & \text { in the framework of Part } 1 \text { of Lemma 2, } \\ {\left[\rho_{n}, 1-\rho_{n}\right]} & \text { in the framework of Part } 2 \text { of Lemma 2, }\end{cases}$

and we prove that

$$
\lim _{n \rightarrow \infty} \sup _{t \in \mathcal{E}_{n}}\left|\Lambda_{n, t}(u)-\frac{u^{2} \sigma^{2}}{2}\right|=0
$$

We first note that

$$
\begin{aligned}
\Lambda_{n, t}(u) & =\frac{v_{n}^{2}}{n h_{n}} \log \mathbb{E}\left[\exp \left(\frac{n h_{n} u\left[\mu_{n}(t)-\mathbb{E}\left(\mu_{n}(t)\right)\right]}{v_{n} \sqrt{I\left(K^{2}, t, h_{n}\right)}}\right)\right] \\
& =\frac{v_{n}^{2}}{n h_{n}} \log \mathbb{E}\left[\exp \left(\frac{u}{v_{n} \sqrt{I\left(K^{2}, t, h_{n}\right)}} \sum_{i=1}^{n}\left[Y_{i} K\left(\frac{t-t_{i}}{h_{n}}\right)-\mathbb{E}\left(Y_{i} K\left(\frac{t-t_{i}}{h_{n}}\right)\right)\right]\right)\right] \\
& =\frac{v_{n}^{2}}{n h_{n}} \log \mathbb{E}\left[\exp \left(\frac{u}{v_{n} \sqrt{I\left(K^{2}, t, h_{n}\right)}} \sum_{i=1}^{n} \varepsilon_{i} K\left(\frac{t-t_{i}}{h_{n}}\right)\right)\right] \\
& =\frac{v_{n}^{2}}{n h_{n}} \sum_{i=1}^{n} \log \mathbb{E}\left[\exp \left(\frac{u}{v_{n} \sqrt{I\left(K^{2}, t, h_{n}\right)}} \varepsilon_{i} K\left(\frac{t-t_{i}}{h_{n}}\right)\right)\right] \\
& =\frac{v_{n}^{2}}{n h_{n}} \sum_{i=1}^{n} \log \left\{1+\mathbb{E}\left[\exp \left(\frac{u}{v_{n} \sqrt{I\left(K^{2}, t, h_{n}\right)}} \varepsilon_{i} K\left(\frac{t-t_{i}}{h_{n}}\right)\right)-1\right]\right\}
\end{aligned}
$$

Now, for each $i \in\{1, \ldots, n\}$, there exists $c_{i, n}$ between 1 and $\mathbb{E}\left[\exp \left(\frac{u}{v_{n} \sqrt{I\left(K^{2}, t, h_{n}\right)}} \varepsilon_{i} K\left(\frac{t-t_{i}}{h_{n}}\right)\right)\right]$ such that

$$
\Lambda_{n, t}(u)=\frac{v_{n}^{2}}{n h_{n}} \sum_{i=1}^{n}\left\{\mathbb{E}\left[\exp \left(\frac{u}{v_{n} \sqrt{I\left(K^{2}, t, h_{n}\right)}} \varepsilon_{i} K\left(\frac{t-t_{i}}{h_{n}}\right)\right)-1\right]+R_{i, n, t}^{(1)}(u)\right\}
$$

where

$$
R_{i, n, t}^{(1)}(u)=\frac{-1}{2 c_{i, n}^{2}}\left\{\mathbb{E}\left[\exp \left(\frac{u}{v_{n} \sqrt{I\left(K^{2}, t, h_{n}\right)}} \varepsilon_{i} K\left(\frac{t-t_{i}}{h_{n}}\right)\right)-1\right]\right\}^{2}
$$


Since $\mathbb{E}\left(\varepsilon_{i}\right)=0$, we get

$$
\Lambda_{n, t}(u)=\frac{v_{n}^{2}}{n h_{n}} \sum_{i=1}^{n}\left\{\mathbb{E}\left[\frac{u^{2}}{2 v_{n}^{2} I\left(K^{2}, t, h_{n}\right)} \varepsilon_{i}^{2} K^{2}\left(\frac{t-t_{i}}{h_{n}}\right)\right]+R_{i, n, t}^{(1)}(u)+R_{i, n, t}^{(2)}(u)\right\}
$$

where

$$
\begin{aligned}
& \left|R_{i, n, t}^{(2)}(u)\right| \\
& \leq \frac{|u|^{3}}{6 v_{n}^{3}\left[I\left(K^{2}, t, h_{n}\right)\right]^{3 / 2}} \mathbb{E}\left[\left|\varepsilon_{i}\right|^{3} K^{3}\left(\frac{t-t_{i}}{h_{n}}\right) \exp \left(\frac{u}{v_{n} \sqrt{I\left(K^{2}, t, h_{n}\right)}}\left|\varepsilon_{i}\right|\|K\|_{\infty}\right)\right] .
\end{aligned}
$$

It follows that

$$
\begin{aligned}
\Lambda_{n, t}(u) & =\frac{u^{2} \sigma^{2}}{2 I\left(K^{2}, t, h_{n}\right)}\left[\frac{1}{n h_{n}} \sum_{i=1}^{n} K^{2}\left(\frac{t-t_{i}}{h_{n}}\right)\right]+\frac{v_{n}^{2}}{n h_{n}} \sum_{i=1}^{n}\left[R_{i, n, t}^{(1)}(u)+R_{i, n, t}^{(2)}(u)\right] \\
& =\frac{u^{2} \sigma^{2}}{2}+\frac{v_{n}^{2}}{n h_{n}} \sum_{i=1}^{n}\left[R_{i, n, t}^{(1)}(u)+R_{i, n, t}^{(2)}(u)\right] .
\end{aligned}
$$

To conclude the proof of Lemma 2, it remains to show that, for $j \in\{1,2\}$,

$$
\lim _{n \rightarrow \infty} \sup _{t \in \mathcal{E}_{n}} \frac{v_{n}^{2}}{n h_{n}} \sum_{i=1}^{n} R_{i, n, t}^{(j)}(u)=0
$$

Let us first note that, by application of Lemma 1, we have

$$
\begin{aligned}
I\left(K^{2}, t, h_{n}\right) & \geq I\left(K^{2}\right)-\sup _{t \in \mathcal{E}_{n}}\left|I\left(K^{2}, t, h_{n}\right)-I\left(K^{2}\right)\right| \\
& \geq \frac{I\left(K^{2}\right)}{2} \text { for } n \text { large enough. }
\end{aligned}
$$

In the same way, we write that

$$
\begin{aligned}
I\left(K^{2}, t, h_{n}\right) & \leq I\left(K^{2}\right)+\sup _{t \in \mathcal{E}_{n}}\left|I\left(K^{2}, t, h_{n}\right)-I\left(K^{2}\right)\right| \\
& \leq 2 I\left(K^{2}\right) \text { for } n \text { large enough. }
\end{aligned}
$$

Let us now look at $\sum_{i=1}^{n} R_{i, n, t}^{(1)}(u)$. Let $c$ denote a generic constant that may vary from line to line. We have, for all $i \in\{1, \ldots, n\}$,

$$
\exp \left(\frac{u}{v_{n} \sqrt{I\left(K^{2}, t, h_{n}\right)}} \varepsilon_{i} K\left(\frac{t-t_{i}}{h_{n}}\right)\right) \geq \exp \left(\frac{-|u|}{v_{n} \sqrt{I\left(K^{2}, t, h_{n}\right)}}\left|\varepsilon_{i}\right|\|K\|_{\infty}\right),
$$

and thus, by (A3), for $n$ large enough,

$$
\begin{aligned}
\frac{1}{c_{i, n}^{2}} & \leq \mathbb{E}\left[\exp \left(\frac{2|u|}{v_{n} \sqrt{I\left(K^{2}, t, h_{n}\right)}}\left|\varepsilon_{i}\right|\|K\|_{\infty}\right)\right] \\
& \leq \mathbb{E}\left[\exp \left(\frac{2|u|}{v_{n} \sqrt{I\left(K^{2}\right) / 2}}\left|\varepsilon_{i}\right|\|K\|_{\infty}\right)\right] \\
& \leq c
\end{aligned}
$$

Journal home page: www.jafristat.net ; www.projecteuclid.org/as 
Moreover, we note that, since $\mathbb{E}\left(\varepsilon_{i}\right)=0$,

$$
\begin{aligned}
& \left|\mathbb{E}\left[\exp \left(\frac{u}{v_{n} \sqrt{I\left(K^{2}, t, h_{n}\right)}} \varepsilon_{i} K\left(\frac{t-t_{i}}{h_{n}}\right)\right)-1\right]\right| \\
& \leq \mathbb{E}\left[\frac{u^{2} \varepsilon_{i}^{2}}{2 v_{n}^{2} I\left(K^{2}, t, h_{n}\right)} K^{2}\left(\frac{t-t_{i}}{h_{n}}\right) \exp \left(\frac{|u|}{v_{n} \sqrt{I\left(K^{2}, t, h_{n}\right)}}\left|\varepsilon_{i}\right|\|K\|_{\infty}\right)\right] \\
& \leq \frac{u^{2}}{2 v_{n}^{2} I\left(K^{2}, t, h_{n}\right)} K^{2}\left(\frac{t-t_{i}}{h_{n}}\right) \mathbb{E}\left[\varepsilon_{i}^{2} \exp \left(\frac{|u|}{v_{n} \sqrt{I\left(K^{2}, t, h_{n}\right)}}\left|\varepsilon_{i}\right|\|K\|_{\infty}\right)\right] \\
& \leq \frac{u^{2}}{v_{n}^{2} I\left(K^{2}\right)} K^{2}\left(\frac{t-t_{i}}{h_{n}}\right) \mathbb{E}\left[\varepsilon _ { i } ^ { 2 } \operatorname { e x p } \left(\frac{|u|}{\left.\left.v_{n} \sqrt{I\left(K^{2}\right) / 2}\left|\varepsilon_{i}\right|\|K\|_{\infty}\right)\right]}\right.\right. \\
& \leq \frac{c}{v_{n}^{2}} K^{2}\left(\frac{t-t_{i}}{h_{n}}\right) .
\end{aligned}
$$

We thus deduce that, for $n$ large enough, and for each $i \in\{1, \ldots, n\}$,

$$
\left|R_{i, n, t}^{(1)}(u)\right| \leq \frac{c}{v_{n}^{4}} K^{4}\left(\frac{t-t_{i}}{h_{n}}\right) .
$$

It follows that, for $n$ large enough,

$$
\begin{aligned}
\left|\frac{v_{n}^{2}}{n h_{n}} \sum_{i=1}^{n} R_{i, n, t}^{(1)}(u)\right| & \leq \frac{c}{v_{n}^{2} n h_{n}} \sum_{i=1}^{n} K^{4}\left(\frac{t-t_{i}}{h_{n}}\right) \\
& \leq \frac{c}{v_{n}^{2}}\left[I\left(K^{4}\right)+\sup _{t \in \mathcal{E}_{n}}\left\{I\left(K^{4}, t, h_{n}\right)-I\left(K^{4}\right)\right\}\right] .
\end{aligned}
$$

Since $v_{n} \rightarrow \infty$, the application of Lemma 1 then ensures that

$$
\lim _{n \rightarrow \infty} \sup _{t \in \mathcal{E}_{n}}\left|\frac{v_{n}^{2}}{n h_{n}} \sum_{i=1}^{n} R_{i, n, t}^{(1)}(u)\right|=0,
$$

so that (7) holds for $j=1$. Let us finally look at $\sum_{i=1}^{n} R_{i, n, t}^{(2)}(u)$. In view of the upper bound (6), we clearly have, for $n$ sufficiently large,

$$
\begin{aligned}
\left|\frac{v_{n}^{2}}{n h_{n}} \sum_{i=1}^{n} R_{i, n, t}^{(2)}(u)\right| & \leq \frac{c}{v_{n} n h_{n}} \sum_{i=1}^{n} K^{3}\left(\frac{t-t_{i}}{h_{n}}\right) \\
& \leq \frac{c}{v_{n}}\left[I\left(K^{3}\right)+\sup _{t \in \mathcal{E}_{n}}\left\{I\left(K^{3}, t, h_{n}\right)-I\left(K^{3}\right)\right\}\right] .
\end{aligned}
$$

The application of Lemma 1 then ensures that

$$
\lim _{n \rightarrow \infty} \sup _{t \in \mathcal{E}_{n}}\left|\frac{v_{n}^{2}}{n h_{n}} \sum_{i=1}^{n} R_{i, n, t}^{(2)}(u)\right|=0,
$$

which proves (7) in the case $j=2$, and concludes the proof of Lemma 2 .

Journal home page: www.jafristat.net ; www.projecteuclid.org/as 


\subsubsection{Proof of Theorem 1}

In view of the first part of Lemma 2, Theorem 1 is a straightforward consequence of the application of Gärtner-Ellis' Theorem (see Dembo and Zeitouni (1998)).

\subsubsection{Proof of Theorem 2}

We first prove the following lemma.

Lemma 3. Under the assumptions of Theorem 2, we have, for any $\delta>0$,

$$
\limsup _{n \rightarrow \infty} \frac{v_{n}^{2}}{n h_{n}} \log \sup _{t \in\left[\rho_{n}, 1-\rho_{n}\right]} \mathbb{P}\left[\frac{v_{n}\left|\mu_{n}(t)-\mathbb{E}\left(\mu_{n}(t)\right)\right|}{\sqrt{I\left(K^{2}, t, h_{n}\right)}} \geq \delta\right] \leq \frac{-\delta^{2}}{2 \sigma^{2}} .
$$

Then, we give the body of the proof of Theorem 2 .

Proof of Lemma 3 For all $u>0$, we have, by application of Chebyshev's inequality,

$$
\begin{aligned}
\mathbb{P}\left[\frac{v_{n}\left(\mu_{n}(t)-\mathbb{E}\left[\mu_{n}(t)\right]\right)}{\sqrt{I\left(K^{2}, t, h_{n}\right)}} \geq \delta\right] & =\mathbb{P}\left[\exp \left(\frac{n h_{n} u\left(\mu_{n}(t)-\mathbb{E}\left[\mu_{n}(t)\right]\right)}{v_{n} \sqrt{I\left(K^{2}, t, h_{n}\right)}}\right) \geq \exp \left(\frac{n h_{n} u \delta}{v_{n}^{2}}\right)\right] \\
& \leq \exp \left[-\frac{n h_{n} u \delta}{v_{n}^{2}}\right] \mathbb{E}\left(\exp \left[\frac{n h_{n} u\left(\mu_{n}(t)-\mathbb{E}\left[\mu_{n}(t)\right]\right)}{v_{n} \sqrt{I\left(K^{2}, t, h_{n}\right)}}\right]\right) \\
& \leq \exp \left[-\frac{n h_{n} u \delta}{v_{n}^{2}}\right] \exp \left[\frac{n h_{n}}{v_{n}^{2}} \Lambda_{n, t}(u)\right],
\end{aligned}
$$

so that

$$
\begin{aligned}
& \frac{v_{n}^{2}}{n h_{n}} \log \sup _{t \in\left[\rho_{n}, 1-\rho_{n}\right]} \mathbb{P}\left[\frac{v_{n}\left(\mu_{n}(t)-\mathbb{E}\left[\mu_{n}(t)\right]\right)}{\sqrt{I\left(K^{2}, t, h_{n}\right)}} \geq \delta\right] \leq-u \delta+\sup _{t \in\left[\rho_{n}, 1-\rho_{n}\right]} \Lambda_{n, t}(u) \\
& \leq-u \delta+\frac{u^{2} \sigma^{2}}{2}+\sup _{t \in\left[\rho_{n}, 1-\rho_{n}\right]}\left|\Lambda_{n, t}(u)-\frac{u^{2} \sigma^{2}}{2}\right| .
\end{aligned}
$$

The application of the second part of Lemma 2 then ensures that, for all $u>0$,

$$
\limsup _{n \rightarrow \infty} \frac{v_{n}^{2}}{n h_{n}} \log \sup _{t \in\left[\rho_{n}, 1-\rho_{n}\right]} \mathbb{P}\left[\frac{v_{n}\left(\mu_{n}(t)-\mathbb{E}\left[\mu_{n}(t)\right]\right)}{\sqrt{I\left(K^{2}, t, h_{n}\right)}} \geq \delta\right] \leq-u \delta+\frac{u^{2} \sigma^{2}}{2} .
$$

It follows that

$$
\begin{aligned}
\limsup _{n \rightarrow \infty} \frac{v_{n}^{2}}{n h_{n}} \log \sup _{t \in\left[\rho_{n}, 1-\rho_{n}\right]} \mathbb{P}\left[\frac{v_{n}\left(\mu_{n}(t)-\mathbb{E}\left[\mu_{n}(t)\right]\right)}{\sqrt{I\left(K^{2}, t, h_{n}\right)}} \geq \delta\right] & \leq \inf _{u>0}\left(-u \delta+\frac{u^{2} \sigma^{2}}{2}\right) \\
& \leq \frac{-\delta^{2}}{2 \sigma^{2}}
\end{aligned}
$$

Journal home page: www.jafristat.net ; www.projecteuclid.org/as 
In the same way, we prove that, for all $u>0$,

$$
\frac{v_{n}^{2}}{n h_{n}} \log \sup _{t \in\left[\rho_{n}, 1-\rho_{n}\right]} \mathbb{P}\left[\frac{v_{n}\left(\mathbb{E}\left[\mu_{n}(t)\right]-\mu_{n}(t)\right)}{\sqrt{I\left(K^{2}, t, h_{n}\right)}} \geq \delta\right] \leq-u \delta+\sup _{t \in\left[\rho_{n}, 1-\rho_{n}\right]} \Lambda_{n, t}(-u),
$$

from which we deduce that

$$
\limsup _{n \rightarrow \infty} \frac{v_{n}^{2}}{n h_{n}} \log \sup _{t \in\left[\rho_{n}, 1-\rho_{n}\right]} \mathbb{P}\left[\frac{v_{n}\left(\mathbb{E}\left[\mu_{n}(t)\right]-\mu_{n}(t)\right)}{\sqrt{I\left(K^{2}, t, h_{n}\right)}} \geq \delta\right] \leq \frac{-\delta^{2}}{2 \sigma^{2}}
$$

Finally, noting that

$$
\begin{gathered}
\mathbb{P}\left[\frac{v_{n}\left|\mu_{n}(t)-\mathbb{E}\left[\mu_{n}(t)\right]\right|}{\sqrt{I\left(K^{2}, t, h_{n}\right)}} \geq \delta\right] \leq \\
2 \max \left\{\mathbb{P}\left[\frac{v_{n}\left(\mu_{n}(t)-\mathbb{E}\left[\mu_{n}(t)\right]\right)}{\sqrt{I\left(K^{2}, t, h_{n}\right)}} \geq \delta\right], \mathbb{P}\left[\frac{v_{n}\left(\mathbb{E}\left[\mu_{n}(t)\right]-\mu_{n}(t)\right)}{\sqrt{I\left(K^{2}, t, h_{n}\right)}} \geq \delta\right]\right\},
\end{gathered}
$$

we obtain

$$
\limsup _{n \rightarrow \infty} \frac{v_{n}^{2}}{n h_{n}} \log \sup _{t \in\left[\rho_{n}, 1-\rho_{n}\right]} \mathbb{P}\left[\frac{v_{n}\left|\mu_{n}(t)-\mathbb{E}\left[\mu_{n}(t)\right]\right|}{\sqrt{I\left(K^{2}, t, h_{n}\right)}} \geq \delta\right] \leq \frac{-\delta^{2}}{2 \sigma^{2}}
$$

Proof of Theorem 2 Set $R_{n}=v_{n}^{-1} h_{n}^{3}$, and let $N(n)$ be the integer part of $R_{n}^{-1}$. There exist $N(n)$ points of $] 0,1\left[, x_{1}^{(n)}, x_{2}^{(n)}, \ldots, x_{N(n)}^{(n)}\right.$, such that

$$
] 0,1\left[\subset \cup_{j=1}^{N(n)} \mathcal{B}_{n}^{(j)} \text { where } \mathcal{B}_{n}^{(j)}=\left\{t \in[0,1],\left|t-x_{j}^{(n)}\right| \leq R_{n}\right\} .\right.
$$

We have

$$
\begin{aligned}
& \mathbb{P}\left[\sup _{t \in\left[\rho_{n}, 1-\rho_{n}\right]} \frac{v_{n}\left|\mu_{n}(t)-\mathbb{E}\left[\mu_{n}(t)\right]\right|}{\sqrt{I\left(K^{2}, t, h_{n}\right)}}\right.\geq \delta] \leq \sum_{j=1}^{N(n)} \mathbb{P}\left[\sup _{t \in \mathcal{B}_{n}^{(j)} \cap\left[\rho_{n}, 1-\rho_{n}\right]} \frac{v_{n}\left|\mu_{n}(t)-\mathbb{E}\left[\mu_{n}(t)\right]\right|}{\sqrt{I\left(K^{2}, t, h_{n}\right)}} \geq \delta\right] \\
& \leq N(n) \max _{1 \leq j \leq N(n)} \mathbb{P}\left[\sup _{t \in \mathcal{B}_{n}^{(j)} \cap\left[\rho_{n}, 1-\rho_{n}\right]} \frac{v_{n}\left|\mu_{n}(t)-\mathbb{E}\left[\mu_{n}(t)\right]\right|}{\sqrt{I\left(K^{2}, t, h_{n}\right)}} \geq \delta\right] .
\end{aligned}
$$

Now, for all $j \in\{1, \ldots, N(n)\}$ and all $t \in \mathcal{B}_{n}^{(j)} \cap\left[\rho_{n}, 1-\rho_{n}\right]$, we have:

$$
\begin{aligned}
\frac{v_{n}\left|\mu_{n}(t)-\mathbb{E}\left[\mu_{n}(t)\right]\right|}{\sqrt{I\left(K^{2}, t, h_{n}\right)}} \leq & \frac{v_{n}\left|\mu_{n}(t)-\mu_{n}\left(x_{j}^{(n)}\right)\right|}{\sqrt{I\left(K^{2}, t, h_{n}\right)}}+\frac{v_{n}\left|\mu_{n}\left(x_{j}^{(n)}\right)-\mathbb{E}\left[\mu_{n}\left(x_{j}^{(n)}\right)\right]\right|}{\sqrt{I\left(K^{2}, t, h_{n}\right)}} \\
& +\frac{v_{n}\left|\mathbb{E}\left[\mu_{n}\left(x_{j}^{(n)}\right)\right]-\mathbb{E}\left[\mu_{n}(t)\right]\right|}{\sqrt{I\left(K^{2}, t, h_{n}\right)}} .
\end{aligned}
$$

Journal home page: www.jafristat.net; www.projecteuclid.org/as 
Set $\eta \in] 0, \delta[$. Since $K$ is Lipschitz, we have, for all $n$ large enough and all $j \in\{1, \ldots, N(n)\}$,

$$
\begin{aligned}
\frac{v_{n}\left|\mathbb{E}\left[\mu_{n}\left(x_{j}^{(n)}\right)\right]-\mathbb{E}\left[\mu_{n}(t)\right]\right|}{\sqrt{I\left(K^{2}, t, h_{n}\right)}} & \leq \frac{v_{n}}{n h_{n} \sqrt{I\left(K^{2}, t, h_{n}\right)}} \sum_{i=1}^{n}\left|\mu\left(t_{i}\right)\right| K\left(\frac{t-t_{i}}{h_{n}}\right)-K\left(\frac{x_{j}^{(n)}-t_{i}}{h_{n}}\right) \mid \\
& \leq \frac{v_{n}\left(\sup _{t \in] 0,1[}|\mu(t)|\right)\|K\|_{L}}{h_{n}^{2} \sqrt{I\left(K^{2}, t, h_{n}\right)}}\left|x_{j}^{(n)}-t\right| \\
& \leq \frac{v_{n}\left(\sup _{t \in] 0,1[}|\mu(t)|\right)\|K\|_{L} R_{n}}{h_{n}^{2} \sqrt{I\left(K^{2}\right) / 2}} \\
& \leq \frac{\eta}{4} .
\end{aligned}
$$

Moreover, we note that, for all $n$ large enough and all $j \in\{1, \ldots, N(n)\},\|K\|_{L} \leq 1 / \sqrt{h_{n}}$, and

$$
\begin{aligned}
\frac{v_{n}\left|\mu_{n}(t)-\mu_{n}\left(x_{j}^{(n)}\right)\right|}{\sqrt{I\left(K^{2}, t, h_{n}\right)}} & \leq \frac{v_{n}}{n h_{n} \sqrt{I\left(K^{2}, t, h_{n}\right)}}\left|\sum_{i=1}^{n} Y_{i}\left[K\left(\frac{t-t_{i}}{h_{n}}\right)-K\left(\frac{x_{j}^{(n)}-t_{i}}{h_{n}}\right)\right]\right| \\
& \leq \frac{v_{n}}{n h_{n} \sqrt{I\left(K^{2}, t, h_{n}\right)}} \sum_{i=1}^{n}\left|Y_{i}\right|\|K\|_{L}\left|\frac{t-x_{j}^{(n)}}{h_{n}}\right| \\
& \leq \frac{v_{n}\|K\|_{L} R_{n}}{n h_{n}^{2} \sqrt{I\left(K^{2}, t, h_{n}\right)}}\left(\sum_{i=1}^{n}\left[\left|\mu\left(t_{i}\right)\right|+\left|\varepsilon_{i}\right|\right]\right) \\
& \leq \frac{v_{n}\|K\|_{L} R_{n}\left(\sup _{t \in] 0,1[}|\mu(t)|\right)}{h_{n}^{2} \sqrt{I\left(K^{2}\right) / 2}}+\frac{v_{n}\|K\|_{L} R_{n}}{n h_{n}^{2} \sqrt{I\left(K^{2}\right) / 2}} \sum_{i=1}^{n}\left|\varepsilon_{i}\right| \\
& \leq \frac{\eta}{4}+\frac{\sqrt{h_{n}}}{n} \sum_{i=1}^{n}\left|\varepsilon_{i}\right|
\end{aligned}
$$

We then deduce that, for all $n$ large enough, all $j \in\{1, \ldots, N(n)\}$, and all $t \in \mathcal{B}_{n}^{(j)} \cap\left[\rho_{n}, 1-\right.$ $\left.\rho_{n}\right]$, we have

$$
\frac{v_{n}\left|\mu_{n}(t)-\mathbb{E}\left[\mu_{n}(t)\right]\right|}{\sqrt{I\left(K^{2}, t, h_{n}\right)}} \leq \frac{v_{n}\left|\mu_{n}\left(x_{j}^{(n)}\right)-\mathbb{E}\left[\mu_{n}\left(x_{j}^{(n)}\right)\right]\right|}{\sqrt{I\left(K^{2}, t, h_{n}\right)}}+\frac{\eta}{2}+\frac{\sqrt{h_{n}}}{n} \sum_{i=1}^{n}\left|\varepsilon_{i}\right|
$$

Journal home page: www.jafristat.net; www.projecteuclid.org/as 
so that, for all $j \in\{1, \ldots, N(n)\}$,

$$
\begin{aligned}
& \mathbb{P}\left[\sup _{t \in \mathcal{B}_{n}^{(j)} \cap\left[\rho_{n}, 1-\rho_{n}\right]} \frac{v_{n}\left|\mu_{n}(t)-\mathbb{E}\left[\mu_{n}(t)\right]\right|}{\sqrt{I\left(K^{2}, t, h_{n}\right)}} \geq \delta\right] \\
& \leq \mathbb{P}\left[\frac{v_{n}\left|\mu_{n}\left(x_{j}^{(n)}\right)-\mathbb{E}\left[\mu_{n}\left(x_{j}^{(n)}\right)\right]\right|}{\sqrt{I\left(K^{2}, t, h_{n}\right)}}+\frac{\sqrt{h_{n}}}{n} \sum_{i=1}^{n}\left|\varepsilon_{i}\right| \geq \delta-\frac{\eta}{2}\right] \\
& \leq \mathbb{P}\left[\frac{v_{n}\left|\mu_{n}\left(x_{j}^{(n)}\right)-\mathbb{E}\left[\mu_{n}\left(x_{j}^{(n)}\right)\right]\right|}{\sqrt{I\left(K^{2}, t, h_{n}\right)}}+\frac{\sqrt{h_{n}}}{n} \sum_{i=1}^{n}\left|\varepsilon_{i}\right| \geq \delta-\frac{\eta}{2} \text { and } \frac{\sqrt{h_{n}}}{n} \sum_{i=1}^{n}\left|\varepsilon_{i}\right|<\frac{\eta}{2}\right] \\
& \quad+\mathbb{P}\left[\frac{\sqrt{h_{n}}}{n} \sum_{i=1}^{n}\left|\varepsilon_{i}\right| \geq \frac{\eta}{2}\right] \\
& \leq \mathbb{P}\left[\frac{v_{n}\left|\mu_{n}\left(x_{j}^{(n)}\right)-\mathbb{E}\left[\mu_{n}\left(x_{j}^{(n)}\right)\right]\right|}{\sqrt{I\left(K^{2}, t, h_{n}\right)}} \geq \delta-\eta\right]+\mathbb{P}\left[\frac{\sqrt{h_{n}}}{n} \sum_{i=1}^{n}\left|\varepsilon_{i}\right| \geq \frac{\eta}{2}\right] .
\end{aligned}
$$

Now,

$$
\mathbb{P}\left[\frac{\sqrt{h_{n}}}{n} \sum_{i=1}^{n}\left|\varepsilon_{i}\right| \geq \frac{\eta}{2}\right]=\mathbb{P}\left[\frac{1}{n} \sum_{i=1}^{n}\left(\left|\varepsilon_{i}\right|-\mathbb{E}\left(\left|\varepsilon_{i}\right|\right)\right) \geq \frac{\eta}{2 \sqrt{h_{n}}}-M_{n}\right]
$$

where $M_{n}=\frac{1}{n} \sum_{i=1}^{n} \mathbb{E}\left(\left|\varepsilon_{i}\right|\right)$. Since the $\varepsilon_{i}$ share the same variance $\sigma^{2}$, we have $M_{n} \leq \sigma$. It follows that, for $n$ large enough, $\frac{\eta}{2 \sqrt{h_{n}}}-M_{n}>\eta$, and thus

$$
\mathbb{P}\left[\frac{\sqrt{h_{n}}}{n} \sum_{i=1}^{n}\left|\varepsilon_{i}\right| \geq \frac{\eta}{2}\right] \leq \mathbb{P}\left[\frac{1}{n} \sum_{i=1}^{n}\left(\left|\varepsilon_{i}\right|-\mathbb{E}\left(\left|\varepsilon_{i}\right|\right)\right) \geq \eta\right]
$$

It follows that, for $n$ large enough,

$$
\begin{aligned}
& \mathbb{P}\left[\sup _{t \in\left[\rho_{n}, 1-\rho_{n}\right]} \frac{v_{n}\left|\mu_{n}(t)-\mathbb{E}\left[\mu_{n}(t)\right]\right|}{\sqrt{I\left(K^{2}, t, h_{n}\right)}} \geq \delta\right] \\
& \leq N(n)\left\{\max _{1 \leq j \leq N(n)} \mathbb{P}\left[\frac{v_{n}\left|\mu_{n}\left(x_{j}^{(n)}\right)-\mathbb{E}\left[\mu_{n}\left(x_{j}^{(n)}\right)\right]\right|}{\sqrt{I\left(K^{2}, t, h_{n}\right)}} \geq \delta-\eta\right]+\mathbb{P}\left[\frac{1}{n} \sum_{i=1}^{n}\left(\left|\varepsilon_{i}\right|-\mathbb{E}\left(\left|\varepsilon_{i}\right|\right)\right) \geq \eta\right]\right\} \\
& \leq N(n)\left\{\sup _{t \in\left[\rho_{n}, 1-\rho_{n}\right]} \mathbb{P}\left[\frac{v_{n}\left|\mu_{n}(t)-\mathbb{E}\left[\mu_{n}(t)\right]\right|}{\sqrt{I\left(K^{2}, t, h_{n}\right)}} \geq \delta-\eta\right]+\mathbb{P}\left[\frac{1}{n} \sum_{i=1}^{n}\left(\left|\varepsilon_{i}\right|-\mathbb{E}\left(\left|\varepsilon_{i}\right|\right)\right) \geq \eta\right]\right\} \\
& \leq 2 N(n) \max \left\{\sup _{t \in\left[\rho_{n}, 1-\rho_{n}\right]} \mathbb{P}\left[\frac{v_{n}\left|\mu_{n}(t)-\mathbb{E}\left[\mu_{n}(t)\right]\right|}{\sqrt{I\left(K^{2}, t, h_{n}\right)}} \geq \delta-\eta\right], \mathbb{P}\left[\frac{1}{n} \sum_{i=1}^{n}\left(\left|\varepsilon_{i}\right|-\mathbb{E}\left(\left|\varepsilon_{i}\right|\right)\right) \geq \eta\right]\right\} .
\end{aligned}
$$


Thus,

$$
\begin{array}{r}
\frac{v_{n}^{2}}{n h_{n}} \log \mathbb{P}\left[\sup _{t \in\left[\rho_{n}, 1-\rho_{n}\right]} \frac{v_{n}\left|\mu_{n}(t)-\mathbb{E}\left[\mu_{n}(t)\right]\right|}{\sqrt{I\left(K^{2}, t, h_{n}\right)}} \geq \delta\right] \\
\leq \frac{v_{n}^{2}}{n h_{n}}\left[\log [2 N(n)]+\max \left\{\log \sup _{t \in\left[\rho_{n}, 1-\rho_{n}\right]} \mathbb{P}\left[\frac{v_{n}\left|\mu_{n}(t)-\mathbb{E}\left[\mu_{n}(t)\right]\right|}{\sqrt{I\left(K^{2}, t, h_{n}\right)}} \geq \delta-\eta\right],\right.\right. \\
\left.\left.\log \mathbb{P}\left[\frac{1}{n} \sum_{i=1}^{n}\left(\left|\varepsilon_{i}\right|-\mathbb{E}\left(\left|\varepsilon_{i}\right|\right)\right) \geq \eta\right]\right\}\right] .
\end{array}
$$

Now, we have:

$$
\lim _{n \rightarrow \infty} \frac{v_{n}^{2}}{n h_{n}} \log [2 N(n)]=\lim _{n \rightarrow \infty} \frac{v_{n}^{2}}{n h_{n}}\left[\log v_{n}+\log \left(1 / h_{n}\right)\right]=0 .
$$

- By application of Lemma 3,

$$
\limsup _{n \rightarrow \infty} \frac{v_{n}^{2}}{n h_{n}} \log \sup _{t \in\left[\rho_{n}, 1-\rho_{n}\right]} \mathbb{P}\left[\frac{v_{n}\left|\mu_{n}(t)-\mathbb{E}\left[\mu_{n}(t)\right]\right|}{\sqrt{I\left(K^{2}, t, h_{n}\right)}} \geq \delta-\eta\right] \leq \frac{-(\delta-\eta)^{2}}{2 \sigma^{2}} .
$$

- By application of Theorem 2.7 of Petrov (1995) to the random variables $\left|\varepsilon_{i}\right|-\mathbb{E}\left(\left|\varepsilon_{i}\right|\right)$,

$\lim _{n \rightarrow \infty} \frac{v_{n}^{2}}{n h_{n}} \log \mathbb{P}\left[\frac{1}{n} \sum_{i=1}^{n}\left(\left|\varepsilon_{i}\right|-\mathbb{E}\left(\left|\varepsilon_{i}\right|\right)\right) \geq \eta\right]=\lim _{n \rightarrow \infty} \frac{v_{n}^{2}}{h_{n}}\left\{\frac{1}{n} \log \mathbb{P}\left[\frac{1}{n} \sum_{i=1}^{n}\left(\left|\varepsilon_{i}\right|-\mathbb{E}\left(\left|\varepsilon_{i}\right|\right)\right) \geq \eta\right]\right\}=-\infty$

We thus deduce that, for any $\eta \in] 0, \delta[$,

$$
\limsup _{n \rightarrow \infty} \frac{v_{n}^{2}}{n h_{n}} \log \mathbb{P}\left[\sup _{t \in\left[\rho_{n}, 1-\rho_{n}\right]} \frac{v_{n}\left|\mu_{n}(t)-\mathbb{E}\left[\mu_{n}(t)\right]\right|}{\sqrt{I\left(K^{2}, t, h_{n}\right)}} \geq \delta\right] \leq \frac{-(\delta-\eta)^{2}}{2 \sigma^{2}} .
$$

Since $\eta>0$ can be chosen arbitrarily small, it follows that

$$
\limsup _{n \rightarrow \infty} \frac{v_{n}^{2}}{n h_{n}} \log \mathbb{P}\left[\sup _{t \in\left[\rho_{n}, 1-\rho_{n}\right]} \frac{v_{n}\left|\mu_{n}(t)-\mathbb{E}\left[\mu_{n}(t)\right]\right|}{\sqrt{I\left(K^{2}, t, h_{n}\right)}} \geq \delta\right] \leq \frac{-\delta^{2}}{2 \sigma^{2}} .
$$

Now, set $t_{0} \in \cap_{n \geq 1}\left[\rho_{n}, 1-\rho_{n}\right]$. We clearly have

$$
\mathbb{P}\left[\sup _{t \in\left[\rho_{n}, 1-\rho_{n}\right]} \frac{v_{n}\left|\mu_{n}(t)-\mathbb{E}\left[\mu_{n}(t)\right]\right|}{\sqrt{I\left(K^{2}, t, h_{n}\right)}} \geq \delta\right] \geq \mathbb{P}\left[\frac{v_{n}\left|\mu_{n}\left(t_{0}\right)-\mathbb{E}\left[\mu_{n}\left(t_{0}\right)\right]\right|}{\sqrt{I\left(K^{2}, t_{0}, h_{n}\right)}} \geq \delta\right]
$$

so that the application of Theorem 1 ensures that

$$
\liminf _{n \rightarrow \infty} \frac{v_{n}^{2}}{n h_{n}} \log \mathbb{P}\left[\sup _{t \in\left[\rho_{n}, 1-\rho_{n}\right]} \frac{v_{n}\left|\mu_{n}(t)-\mathbb{E}\left[\mu_{n}(t)\right]\right|}{\sqrt{I\left(K^{2}, t, h_{n}\right)}} \geq \delta\right] \geq \frac{-\delta^{2}}{2 \sigma^{2}} .
$$

The combination of (8) and (9) gives

$$
\lim _{n \rightarrow \infty} \frac{v_{n}^{2}}{n h_{n}} \log \mathbb{P}\left[\sup _{t \in\left[\rho_{n}, 1-\rho_{n}\right]} \frac{v_{n}\left|\mu_{n}(t)-\mathbb{E}\left[\mu_{n}(t)\right]\right|}{\sqrt{I\left(K^{2}, t, h_{n}\right)}} \geq \delta\right]=-J(\delta) .
$$

Journal home page: www.jafristat.net ; www.projecteuclid.org/as 
Since the sequence $\left(\sup _{t \in\left[\rho_{n}, 1-\rho_{n}\right]} \frac{v_{n}\left|\mu_{n}(t)-\mathbb{E}\left[\mu_{n}(t)\right]\right|}{\sqrt{I\left(K^{2}, t, h_{n}\right)}}\right)$ is positive and since $J$ is continuous on $[0,+\infty[$, increasing, and goes to $\infty$ as $\delta \rightarrow \infty$, the application of Lemma 5 in Worms (2001) ensures that $\left(\sup _{t \in\left[\rho_{n}, 1-\rho_{n}\right]} \frac{v_{n}\left|\mu_{n}(t)-\mathbb{E}\left[\mu_{n}(t)\right]\right|}{\sqrt{I\left(K^{2}, t, h_{n}\right)}}\right)$ satisfies a LDP with speed $\left(n h_{n} / v_{n}^{2}\right)$ and good rate function $J$ on $\mathbb{R}^{+}$.

\subsection{Proof of Corollaries 1 and 2}

Corollary 1 (respectively Corollary 2) is a straightforward consequence of Theorem 1 (respectively Theorem 2) together with the following lemma.

Lemma 4. 1) Under the assumptions of Corollary 1, we have, $\forall t \in] 0,1[$,

$$
\lim _{n \rightarrow \infty} v_{n}\left|\mathbb{E}\left(\mu_{n}(t)\right)-\mu(t)\right|=0 .
$$

2) Under the assumptions of Corollary 2, we have

$$
\lim _{n \rightarrow \infty} \sup _{t \in\left[\rho_{n}, 1-\rho_{n}\right]} v_{n}\left|\mathbb{E}\left(\mu_{n}(t)\right)-\mu(t)\right|=0 .
$$

In order to give an unified proof for both parts of Lemma 4, we set again

$$
\mathcal{E}_{n}= \begin{cases}\{t\} & \text { in the framework of Part } 1 \text { of Lemma } 4, \\ {\left[\rho_{n}, 1-\rho_{n}\right]} & \text { in the framework of Part } 2 \text { of Lemma } 4,\end{cases}
$$

and we prove that

$$
\lim _{n \rightarrow \infty} \sup _{t \in \mathcal{E}_{n}} v_{n}\left|\mathbb{E}\left(\mu_{n}(t)\right)-\mu(t)\right|=0 .
$$

Let $c$ denote a generic constant that may vary from line to line. We first note that, $\forall t \in \mathcal{E}_{n}$,

$$
\begin{aligned}
& \left|\mathbb{E}\left(\mu_{n}(t)\right)-\frac{1}{h_{n}} \int_{0}^{1} \mu(s) K\left(\frac{t-s}{h_{n}}\right) d s\right| \\
& =\left|\frac{1}{n h_{n}} \sum_{i=1}^{n} \mu\left(t_{i}\right) K\left(\frac{t-t_{i}}{h_{n}}\right)-\frac{1}{h_{n}} \int_{0}^{1} \mu(s) K\left(\frac{t-s}{h_{n}}\right) d s\right| \\
& \leq \frac{1}{h_{n}} \sum_{i=1}^{n} \int_{t_{i}}^{t_{i+1}}\left|\mu\left(t_{i}\right) K\left(\frac{t-t_{i}}{h_{n}}\right)-\mu(s) K\left(\frac{t-s}{h_{n}}\right)\right| d s \\
& \leq \frac{1}{h_{n}} \sum_{i=1}^{n}\left\{\int_{t_{i}}^{t_{i+1}}\left|\mu\left(t_{i}\right)-\mu(s)\right| K\left(\frac{t-t_{i}}{h_{n}}\right) d s+\int_{t_{i}}^{t_{i+1}}|\mu(s)| K\left(\frac{t-t_{i}}{h_{n}}\right)-K\left(\frac{t-s}{h_{n}}\right) \mid d s\right\} \\
& \leq \frac{\|K\|_{\infty}}{h_{n}} \sum_{i=1}^{n} \int_{t_{i}}^{t_{i+1}}\|\mu\|_{L}\left(s-t_{i}\right) d s+\frac{\left.\sup _{t \in] 0,1[}|\mu(t)|\right)}{h_{n}^{2}} \sum_{i=1}^{t_{i+1}} \int_{t_{i}}\|K\|_{L}\left(s-t_{i}\right) d s \\
& \leq \frac{\|K\|_{\infty}\|\mu\|_{L}}{2 n h_{n}}+\frac{\left(\sup _{t \in] 0,1[}|\mu(t)|\right)\|K\|_{L}}{2 n h_{n}^{2}} \\
& \leq \frac{c}{n h_{n}^{2}} .
\end{aligned}
$$

Journal home page: www.jafristat.net ; www.projecteuclid.org/as 
Now, in the framework of the first part of Lemma 4 , we introduce a sequence $\left(\rho_{n}\right)$ such that

$$
\lim _{n \rightarrow \infty} \rho_{n}=0 \text { and } \rho_{n} \geq h_{n}^{(m-3) /(m-1)} \text { for all } n
$$

(where $m$ is defined in $(A 1) i i)$ ). We then have

$$
\lim _{n \rightarrow \infty} \frac{\rho_{n}}{h_{n}}=\infty, \text { and } t \in\left[\rho_{n}, 1-\rho_{n}\right] \text { for all } n \text { large enough. }
$$

We deduce that, for $n$ large enough and $\forall t \in \mathcal{E}_{n}$,

$$
\begin{aligned}
& \left|\frac{1}{h_{n}} \int_{0}^{1} \mu(s) K\left(\frac{t-s}{h_{n}}\right) d s-\mu(t)\right| \\
& =\left|\int_{(t-1) / h_{n}}^{t / h_{n}} \mu\left(t-h_{n} u\right) K(u) d u-\int_{\mathbb{R}} \mu(t) K(u) d u\right| \\
& \leq\left|\int_{(t-1) / h_{n}}^{t / h_{n}}\left[\mu\left(t-h_{n} u\right)-\mu(t)\right] K(u) d u\right|+\left(\sup _{t \in] 0,1[}|\mu(t)|\right)\left|\int_{-\infty}^{(t-1) / h_{n}} K(u) d u+\int_{t / h_{n}}^{\infty} K(u) d u\right| \\
& \leq\left|\int_{(t-1) / h_{n}}^{t / h_{n}}\left[\mu\left(t-h_{n} u\right)-\mu(t)\right] K(u) d u\right|+\left(\sup _{t \in] 0,1[}|\mu(t)|\right) \int_{|u|>\rho_{n} / h_{n}} K(u) d u .
\end{aligned}
$$

Since $\lim _{u \rightarrow \infty}|u|^{m} K(u) d u=0$ and $\lim _{n \rightarrow \infty} \rho_{n} / h_{n}=\infty$, we have, for $n$ large enough and $\forall t \in \mathcal{E}_{n}$,

$$
\begin{aligned}
\int_{|u|>\rho_{n} / h_{n}} K(u) d u & \leq \int_{|u|>\rho_{n} / h_{n}}|u|^{-m} d u \\
& \leq c\left(\frac{\rho_{n}}{h_{n}}\right)^{1-m} \\
& \leq c h_{n}^{2} \text { since } \rho_{n} \geq h_{n}^{(m-3) /(m-1)}
\end{aligned}
$$

It follows that, for $n$ large enough and $\forall t \in \mathcal{E}_{n}$,

$$
\left|\mathbb{E}\left(\mu_{n}(t)\right)-\mu(t)\right| \leq\left|\int_{(t-1) / h_{n}}^{t / h_{n}}\left[\mu\left(t-h_{n} u\right)-\mu(t)\right] K(u) d u\right|+c\left[\frac{1}{n h_{n}^{2}}+h_{n}^{2}\right] .
$$

Now, since $\int_{(t-1) / h_{n}}^{t / h_{n}} u K(u) d u=-\int_{u \notin\left[\frac{t-1}{h_{n}}, \frac{t}{h_{n}}\right]} u K(u) d u$ and since $\mu$ is twice differentiable at $t$, we have

$$
\begin{aligned}
& \int_{(t-1) / h_{n}}^{t / h_{n}}\left[\mu\left(t-h_{n} u\right)-\mu(t)\right] K(u) d u \\
& =h_{n}^{2} \int_{\mathbb{R}} u^{2}\left[\frac{\mu\left(t-h_{n} u\right)-\mu(t)+h_{n} u \mu^{\prime}(t)}{h_{n}^{2} u^{2}} \mathbb{1}_{\left[\frac{t-1}{h_{n}}, \frac{t}{h_{n}}\right]}\right] K(u) d u \\
& \quad+h_{n} \mu^{\prime}(t) \int_{u \notin\left[\frac{t-1}{h_{n}}, \frac{t}{h_{n}}\right]} u K(u) d u .
\end{aligned}
$$


It is straightforward that the second right-handed term is $O\left(h_{n}^{4}\right)$. For the first right-handed term, we note that, for any $u \neq 0$,

$$
\lim _{n \rightarrow \infty}\left[\frac{\mu\left(t-h_{n} u\right)-\mu(t)+h_{n} u \mu^{\prime}(t)}{h_{n}^{2} u^{2}} \mathbb{1}_{\left[\frac{t-1}{h_{n}}, \frac{t}{h_{n}}\right]}\right]=\frac{\mu^{\prime \prime}(t)}{2} .
$$

In the framework of the first part of Lemma 4, it can easily be seen that the bracketed term in (11) is bounded for each $t$ by a constant independent of $h_{n}$ and $u$. It follows that, by Lebesgue's Theorem,

$$
\lim _{n \rightarrow \infty} \frac{1}{h_{n}^{2}} \int_{(t-1) / h_{n}}^{t / h_{n}}\left[\mu\left(t-h_{n} u\right)-\mu(t)\right] K(u) d u=\frac{\mu^{\prime \prime}(t)}{2} \int_{\mathbb{R}} u^{2} K(u) d u .
$$

In the framework of the second part of Lemma 4, we note that, in view of Taylor expansion, the bracketed term in $(11)$ is uniformly bounded by $\left(\sup _{t \in] 0,1[}\left|\mu^{\prime \prime}(t)\right|\right)$, and thus

$$
\limsup _{n \rightarrow \infty} \frac{1}{h_{n}^{2}} \sup _{t \in\left[\rho_{n}, 1-\rho_{n}\right]}\left|\int_{(t-1) / h_{n}}^{t / h_{n}}\left[\mu\left(t-h_{n} u\right)-\mu(t)\right] K(u) d u\right|<+\infty .
$$

In both cases, we finally obtain

$$
\sup _{t \in \mathcal{E}_{n}} v_{n}\left|\mathbb{E}\left(\mu_{n}(t)\right)-\mu(t)\right| \leq c v_{n}\left(h_{n}^{2}+\frac{1}{n h_{n}^{2}}\right) .
$$

Since $v_{n} h_{n}^{2} \rightarrow 0$ and $v_{n} /\left(n h_{n}^{2}\right) \rightarrow 0$, Lemma 4 follows.

Remark: If the support of $K$ is bounded, then, the integrals $\int_{|u|>\rho_{n} / h_{n}} K(u) d u$ and $\int_{|u|>\rho_{n} / h_{n}}|u| K(u) d u$ vanish for $n$ large enough, so that, for $n$ sufficiently large,

$$
\sup _{t \in \mathcal{E}_{n}}\left|\mathbb{E}\left(\mu_{n}(t)\right)-\mu(t)\right| \leq c t e\left[\frac{1}{n h_{n}^{2}}+h_{n}^{2}\right]
$$

without any additional condition on $\rho_{n}$.

\subsection{Proof of Propositions 1 and 2, and of Corollaries 3 and 4}

We first show how the logarithmic asymptotic levels given in the first parts of Propositions 1 and 2 (and of Corollaries 3 and 4) are deduced from the MDP stated in Corollaries 1 and 2. Then, we establish the almost sure parts of our results stated in the second parts of Propositions 1 and 2 (and of Corollaries 3 and 4).

\subsubsection{Logarithmic asymptotic levels}

We need the following preliminary lemma.

Lemma 5. Assume $\left(A^{\prime} 3\right)$ holds. Then, for all $\delta>0$, there exists $c(\delta)>0$ such that

$$
\limsup _{n \rightarrow \infty} \frac{1}{n} \log \mathbb{P}\left(\left|\hat{\sigma}_{n}^{2}-\sigma^{2}\right| \geq \delta\right) \leq-c(\delta) .
$$


Proof of Lemma 5

For $i \in\{2, \ldots, n-1\}$, set

$$
U_{i}=\frac{2}{3}\left(\frac{1}{2} Y_{i-1}+\frac{1}{2} Y_{i+1}-Y_{i}\right)^{2}-\mathbb{E}\left[\frac{2}{3}\left(\frac{1}{2} Y_{i-1}+\frac{1}{2} Y_{i+1}-Y_{i}\right)^{2}\right] .
$$

We have

$$
\mathbb{P}\left(\left|\hat{\sigma}_{n}^{2}-\mathbb{E}\left(\hat{\sigma}_{n}^{2}\right)\right| \geq \delta\right) \leq \mathbb{P}_{1}+\mathbb{P}_{2}+\mathbb{P}_{3}
$$

with

$$
\begin{aligned}
& \mathbb{P}_{1}=\mathbb{P}\left(\frac{1}{n-2}\left|\sum_{\substack{j=2+3 i \\
0 \leq i \leq(n-4) / 3}} U_{j}\right| \geq \frac{\delta}{3}\right) \\
& \mathbb{P}_{2}=\mathbb{P}\left(\frac{1}{n-2}\left|\sum_{\substack{j=3+3 i \\
0 \leq i \leq(n-5) / 3}} U_{j}\right| \geq \frac{\delta}{3}\right) \\
& \mathbb{P}_{3}=\mathbb{P}\left(\frac{1}{n-2}\left|\sum_{\substack{j=4+3 i \\
0 \leq i \leq(n-6) / 3}} U_{j}\right| \geq \frac{\delta}{3}\right)
\end{aligned}
$$

In view of Assumption $\left(A^{\prime} 3\right)$ and Lemma 2.2 of Petrov (1995), easy computations ensure the existence of $\gamma>0$ and $T>0$ such that, for all $i \in\{2, \ldots, n-1\}$ and for all $t \in[-T, T]$, $\mathbb{E}\left(\exp \left(t U_{i}\right)\right)<\exp \left(g t^{2}\right)$. Since the random variables that appear in the sum of $\mathbb{P}_{k}(k \in$ $\{1,2,3\})$ are independent, Theorem 2.7 of Petrov (1995) applies and ensures that

$$
\mathbb{P}_{k} \leq 2 \max \left\{\exp \left(\frac{-\delta^{2}(n-2)^{2}}{9 n \gamma}\right), \exp \left(\frac{-T \delta(n-2)}{6}\right)\right\}
$$

It follows that

$$
\begin{aligned}
\limsup _{n \rightarrow \infty} \frac{1}{n} \log \mathbb{P}\left(\left|\hat{\sigma}_{n}^{2}-\mathbb{E}\left(\hat{\sigma}_{n}^{2}\right)\right| \geq \delta\right) & \leq \limsup _{n \rightarrow \infty} \frac{1}{n}\left[\log 3+\log \left(\max _{1 \leq k \leq 3} \mathbb{P}_{k}\right)\right] \\
& \leq \max \left\{\frac{-\delta^{2}}{9 \gamma}, \frac{-T \delta}{6}\right\} .
\end{aligned}
$$

Setting $c(\delta)=\min \left\{\delta^{2} /(9 \gamma), T \delta / 6\right\}$, we get

$$
\limsup _{n \rightarrow \infty} \frac{1}{n} \log \mathbb{P}\left(\left|\hat{\sigma}_{n}^{2}-\mathbb{E}\left(\hat{\sigma}_{n}^{2}\right)\right| \geq \delta\right) \leq-c(\delta) .
$$

Since $\hat{\sigma}_{n}^{2}$ is asymptotically unbiased, Lemma 5 follows.

Journal home page: www.jafristat.net ; www.projecteuclid.org/as 
Proof of the first part of Propositions 1 and 2, and of Corollaries 3 and 4

In order to give a unified proof of Propositions 1 and 2, and of Corollaries 3 and 4, we introduce the following notations.

$$
\mathcal{E}_{n}= \begin{cases}\{t\} & \text { in the framework of Proposition } 1 \text { and of Corollary } 3 \\ {\left[\rho_{n}, 1-\rho_{n}\right]} & \text { in the framework of Proposition } 2 \text { and of Corollary } 4\end{cases}
$$

$A_{n}(t)= \begin{cases}I\left(K^{2}, t, h_{n}\right) & \text { in the framework of Propositions } 1 \text { and } 2 \\ I\left(K^{2}, t, h_{n}^{*}\right) \text { or } I\left(K^{2}\right) & \text { in the framework of Corollaries } 3 \text { and } 4\end{cases}$

With these notations, the first part of Propositions 1 and 2, and of Corollaries 3 and 4 can be rewritten as

$$
\lim _{n \rightarrow \infty} \frac{v_{n}^{2}}{n h_{n}} \log \mathbb{P}\left(\exists t \in \mathcal{E}_{n}, \mu(t) \notin\left[\mu_{n}(t)-\delta \frac{\hat{\sigma}_{n} \sqrt{A_{n}(t)}}{v_{n}}, \mu_{n}(t)+\delta \frac{\hat{\sigma}_{n} \sqrt{A_{n}(t)}}{v_{n}}\right]\right)=-\frac{\delta^{2}}{2} .
$$

Set $\delta>0$ and $\eta \in] 0,1[$. We have

$$
\begin{aligned}
& \mathbb{P}\left(\exists t \in \mathcal{E}_{n}, \mu(t) \notin\left[\mu_{n}(t)-\delta \frac{\hat{\sigma}_{n} \sqrt{A_{n}(t)}}{v_{n}}, \mu_{n}(t)+\delta \frac{\hat{\sigma}_{n} \sqrt{A_{n}(t)}}{v_{n}}\right]\right) \\
& =\mathbb{P}\left[\sup _{t \in \mathcal{E}_{n}} \frac{v_{n}\left|\mu_{n}(t)-\mu(t)\right|}{\sqrt{A_{n}(t)}}>\delta \hat{\sigma}_{n}\right] \\
& \leq \mathbb{P}\left[\sup _{t \in \mathcal{E}_{n}} \frac{v_{n}\left|\mu_{n}(t)-\mu(t)\right|}{\left.\sqrt{A_{n}(t)} \geq \delta \hat{\sigma}_{n} \text { and } \hat{\sigma}_{n}^{2} \geq(1-\eta) \sigma^{2}\right]+\mathbb{P}\left[\hat{\sigma}_{n}^{2}<(1-\eta) \sigma^{2}\right]}\right. \\
& \leq \mathbb{P}\left[\sup _{t \in \mathcal{E}_{n}} \frac{v_{n}\left|\mu_{n}(t)-\mu(t)\right|}{\sigma \sqrt{A_{n}(t)}} \geq \delta \sqrt{1-\eta}\right]+\mathbb{P}\left[\hat{\sigma}_{n}^{2}-\sigma^{2}<-\eta \sigma^{2}\right] \\
& \leq \mathbb{P}\left[\left(\sup _{t \in \mathcal{E}_{n}} \frac{\sqrt{I\left(K^{2}, t, h_{n}\right)}}{\sqrt{A_{n}(t)}}\right)\left(\sup _{t \in \mathcal{E}_{n}} \frac{v_{n}\left|\mu_{n}(t)-\mu(t)\right|}{\sigma \sqrt{I\left(K^{2}, t, h_{n}\right)}}\right) \geq \delta \sqrt{1-\eta}\right]+\mathbb{P}\left[\left|\hat{\sigma}_{n}^{2}-\sigma^{2}\right|>\eta \sigma^{2}\right] .
\end{aligned}
$$

For $n$ sufficiently large, we have

$$
\sup _{t \in \mathcal{E}_{n}} \frac{\sqrt{I\left(K^{2}, t, h_{n}\right)}}{\sqrt{A_{n}(t)}} \leq \sqrt{1+\eta},
$$

(this is obvious in the framework of Propositions 1 and 2, and a consequence of Lemma 1 in the framework of Corollaries 3 and 4). It follows that, for $n$ large enough,

$$
\begin{aligned}
& \mathbb{P}\left(\exists t \in \mathcal{E}_{n}, \mu(t) \notin\left[\mu_{n}(t)-\delta \frac{\hat{\sigma}_{n} \sqrt{A_{n}(t)}}{v_{n}}, \mu_{n}(t)+\delta \frac{\hat{\sigma}_{n} \sqrt{A_{n}(t)}}{v_{n}}\right]\right) \\
& \leq \mathbb{P}\left[\sup _{t \in \mathcal{E}_{n}} \frac{v_{n}\left|\mu_{n}(t)-\mu(t)\right|}{\sigma \sqrt{I\left(K^{2}, t, h_{n}\right)}} \geq \frac{\delta \sqrt{1-\eta}}{\sqrt{1+\eta}}\right]+\mathbb{P}\left[\left|\hat{\sigma}_{n}^{2}-\sigma^{2}\right|>\eta \sigma^{2}\right], \\
& \leq 2 \max \left\{\mathbb{P}\left[\sup _{t \in \mathcal{E}_{n}} \frac{v_{n}\left|\mu_{n}(t)-\mu(t)\right|}{\sigma \sqrt{I\left(K^{2}, t, h_{n}\right)}} \geq \frac{\delta \sqrt{1-\eta}}{\sqrt{1+\eta}}\right], \mathbb{P}\left[\left|\hat{\sigma}_{n}^{2}-\sigma^{2}\right|>\eta \sigma^{2}\right]\right\} .
\end{aligned}
$$

Journal home page: www.jafristat.net ; www.projecteuclid.org/as 
Now, the application of Corollary 1 (respectively of Corollary 2) in the framework of Proposition 1 and Corollary 3 (respectively of Proposition 2 and Corollary 4) ensures that

$$
\lim _{n \rightarrow \infty} \frac{v_{n}^{2}}{n h_{n}} \log \mathbb{P}\left[\sup _{t \in \mathcal{E}_{n}} \frac{v_{n}\left|\mu_{n}(t)-\mu(t)\right|}{\sigma \sqrt{I\left(K^{2}, t, h_{n}\right)}} \geq \frac{\delta \sqrt{1-\eta}}{\sqrt{1+\eta}}\right]=\frac{-\delta^{2}(1-\eta)}{2(1+\eta)} .
$$

The application of Lemma 5 giving

$$
\begin{aligned}
\limsup _{n \rightarrow \infty} \frac{v_{n}^{2}}{n h_{n}} \log \mathbb{P}\left[\left|\hat{\sigma}_{n}^{2}-\sigma^{2}\right|>\eta \sigma^{2}\right] & \leq\left(\limsup _{n \rightarrow \infty} \frac{v_{n}^{2}}{h_{n}}\right)\left[-c\left(\eta \sigma^{2}\right)\right] \\
& =-\infty
\end{aligned}
$$

we deduce that

$$
\begin{aligned}
\limsup _{n \rightarrow \infty} \frac{v_{n}^{2}}{n h_{n}} \log \mathbb{P}\left(\exists t \in \mathcal{E}_{n}, \mu(t) \notin\right. & {\left[\mu_{n}(t)-\delta \frac{\hat{\sigma}_{n} \sqrt{A_{n}(t)}}{v_{n}},\right.} \\
& \left.\left.\mu_{n}(t)+\delta \frac{\hat{\sigma}_{n} \sqrt{A_{n}(t)}}{v_{n}}\right]\right) \leq \frac{-\delta^{2}(1-\eta)}{2(1+\eta)} .
\end{aligned}
$$

Since $\eta \in] 0,1[$ can be chosen arbitrarily small, we obtain

$$
\begin{aligned}
\limsup _{n \rightarrow \infty} \frac{v_{n}^{2}}{n h_{n}} \log \mathbb{P}\left(\exists t \in \mathcal{E}_{n}, \mu(t) \notin\right. & {\left[\mu_{n}(t)-\delta \frac{\hat{\sigma}_{n} \sqrt{A_{n}(t)}}{v_{n}},\right.} \\
& \left.\left.\mu_{n}(t)+\delta \frac{\hat{\sigma}_{n} \sqrt{A_{n}(t)}}{v_{n}}\right]\right) \leq \frac{-\delta^{2}}{2} .
\end{aligned}
$$

On the other hand, set $t_{0} \in \cap_{n \geq 1} \mathcal{E}_{n}$. For $n$ large enough, we have $I\left(K^{2}, t_{0}, h_{n}\right) / A_{n}\left(t_{0}\right)>$ $(1-\eta)$, and thus

$$
\begin{aligned}
& \mathbb{P}\left(\exists t \in \mathcal{E}_{n}, \mu(t) \notin\left[\mu_{n}(t)-\delta \frac{\hat{\sigma}_{n} \sqrt{A_{n}(t)}}{v_{n}}, \mu_{n}(t)+\delta \frac{\hat{\sigma}_{n} \sqrt{A_{n}(t)}}{v_{n}}\right]\right) \\
& =\mathbb{P}\left[\sup _{t \in \mathcal{E}_{n}} \frac{v_{n}\left|\mu_{n}(t)-\mu(t)\right|}{\left.\sqrt{A_{n}(t)}>\delta \hat{\sigma}_{n}\right]}\right. \\
& \geq \mathbb{P}\left[\frac{v_{n}\left|\mu_{n}\left(t_{0}\right)-\mu\left(t_{0}\right)\right|}{\left.\sqrt{A_{n}\left(t_{0}\right)}>\delta \hat{\sigma}_{n}\right]}\right. \\
& \geq \mathbb{P}\left[\frac{v_{n}\left|\mu_{n}\left(t_{0}\right)-\mu\left(t_{0}\right)\right|}{\left.\sqrt{I\left(K^{2}, t_{0}, h_{n}\right)}>\frac{\delta \sigma}{1-\eta} \text { and } \sigma^{2}>(1-\eta) \hat{\sigma}_{n}^{2}\right]}\right. \\
& \geq \mathbb{P}\left[\frac{v_{n}\left|\mu_{n}\left(t_{0}\right)-\mu\left(t_{0}\right)\right|}{\sigma \sqrt{I\left(K^{2}, t_{0}, h_{n}\right)}}>\frac{\delta}{1-\eta}\right]-\mathbb{P}\left[\sigma^{2} \leq(1-\eta) \hat{\sigma}_{n}^{2}\right] \\
& \geq \mathbb{P}\left[\frac{v_{n}\left|\mu_{n}\left(t_{0}\right)-\mu\left(t_{0}\right)\right|}{\sigma \sqrt{I\left(K^{2}, t_{0}, h_{n}\right)}}>\frac{\delta}{1-\eta}\right]-\mathbb{P}\left[\left|\hat{\sigma}_{n}^{2}-\sigma^{2}\right| \geq \frac{\eta \sigma^{2}}{1-\eta}\right] .
\end{aligned}
$$

Journal home page: www.jafristat.net; www.projecteuclid.org/as 
Now, the application of Corollary 1 ensures that

$$
\lim _{n \rightarrow \infty} \frac{v_{n}^{2}}{n h_{n}} \log \mathbb{P}\left[\frac{v_{n}\left|\mu_{n}\left(t_{0}\right)-\mu\left(t_{0}\right)\right|}{\sigma \sqrt{I\left(K^{2}, t_{0}, h_{n}\right)}}>\frac{\delta}{1-\eta}\right]=\frac{-\delta^{2}}{2(1-\eta)^{2}} .
$$

Since, in view of Lemma 5 , we have

$$
\limsup _{n \rightarrow \infty} \frac{1}{n} \log \mathbb{P}\left[\left|\hat{\sigma}_{n}^{2}-\sigma^{2}\right| \geq \frac{\eta \sigma^{2}}{1-\eta}\right] \leq-c\left(\frac{\eta \sigma^{2}}{1-\eta}\right),
$$

we deduce from (16) that

$$
\lim _{n \rightarrow \infty} \frac{\mathbb{P}\left[\left|\hat{\sigma}_{n}^{2}-\sigma^{2}\right| \geq \frac{\eta \sigma^{2}}{1-\eta}\right]}{\mathbb{P}\left[\frac{v_{n}\left|\mu_{n}\left(t_{0}\right)-\mu\left(t_{0}\right)\right|}{\sigma \sqrt{I\left(K^{2}, t_{0}, h_{n}\right)}}>\frac{\delta}{1-\eta}\right]}=0,
$$

so that, for sufficiently large $n$, we obtain

$$
\begin{aligned}
\mathbb{P}\left(\exists t \in \mathcal{E}_{n}, \mu(t) \notin\left[\mu_{n}(t)-\right.\right. & \left.\left.\delta \frac{\hat{\sigma}_{n} \sqrt{A_{n}(t)}}{v_{n}}, \mu_{n}(t)+\delta \frac{\hat{\sigma}_{n} \sqrt{A_{n}(t)}}{v_{n}}\right]\right) \\
& \geq \frac{1}{2} \mathbb{P}\left[\frac{v_{n}\left|\mu_{n}\left(t_{0}\right)-\mu\left(t_{0}\right)\right|}{\sigma \sqrt{I\left(K^{2}, t_{0}, h_{n}\right)}}>\frac{\delta}{1-\eta}\right] .
\end{aligned}
$$

Applying (16) again, we get

$$
\begin{aligned}
\liminf _{n \rightarrow \infty} \frac{v_{n}^{2}}{n h_{n}} \log \mathbb{P}\left(\exists t \in \mathcal{E}_{n}, \mu(t) \notin[\right. & \mu_{n}(t)-\delta \frac{\hat{\sigma}_{n} \sqrt{A_{n}(t)}}{v_{n}}, \\
& \left.\left.\mu_{n}(t)+\delta \frac{\hat{\sigma}_{n} \sqrt{A_{n}(t)}}{v_{n}}\right]\right) \geq \frac{-\delta^{2}}{2(1-\eta)^{2}},
\end{aligned}
$$

and thus, since $\eta \in] 0,1[$ can be chosen arbitrarily small,

$$
\begin{aligned}
\liminf _{n \rightarrow \infty} \frac{v_{n}^{2}}{n h_{n}} \log \mathbb{P}\left(\exists t \in \mathcal{E}_{n}, \mu(t) \notin[\right. & \mu_{n}(t)-\delta \frac{\hat{\sigma}_{n} \sqrt{A_{n}(t)}}{v_{n}}, \\
& \left.\left.\mu_{n}(t)+\delta \frac{\hat{\sigma}_{n} \sqrt{A_{n}(t)}}{v_{n}}\right]\right) \geq \frac{-\delta^{2}}{2} .
\end{aligned}
$$

Property (14), and thus the first part of Propositions 1 and 2, and of Corollaries 3 and 4, are then a straightforward combination of (15) and (17).

\subsubsection{Asymptotic almost sure confidence regions}

Set

$$
D_{n}=\left\{g:[0,1] \rightarrow \mathbb{R},\left|g(t)-\mu_{n}(t)\right| \leq \delta \frac{\hat{\sigma}_{n} \sqrt{A_{n}(t)}}{v_{n}} \forall t \in \mathcal{E}_{n}\right\}
$$


where $\mathcal{E}_{n}$ and $A_{n}(t)$ are defined in (12) and (13) respectively. The application of (14) ensures that the logarithmic asymptotic level of $\left\{D_{n}\right\}$ is $\delta^{2} / 2$ with speed $n h_{n} / v_{n}^{2}$. Set $\left.\gamma \in\right] 0, \delta^{2} / 2[$. In view of Proposition 1.3 in Mokkadem and Pelletier (2006), if

$$
\sum \exp \left(-\gamma \frac{n h_{n}}{v_{n}^{2}}\right)<\infty
$$

then $\left\{D_{n}\right\}$ is an asymptotic almost sure sequence of confidence regions. To prove (18), we use the conditions

$$
v_{n} h_{n}^{2} \rightarrow 0, \text { and } \frac{n h_{n}}{v_{n}^{2} \log \left(1 / h_{n}\right)} \rightarrow \infty
$$

(the first one is in the statement of Propositions 1 and 2, the second one is the additional condition in Proposition 1, and stands in Conditions (3) required in Proposition 2). Set $\eta \in] 0,1 / 5\left[\right.$. On the one hand, the first condition in (19) ensures that $n h_{n} v_{n}^{-2}\left[n h_{n}^{5}\right]^{-1} \rightarrow \infty$, and thus, for $n$ large enough,

$$
\begin{aligned}
\exp \left(-\gamma \frac{n h_{n}}{v_{n}^{2}}\right) & \leq \exp \left(-\gamma n h_{n}^{5}\right) \\
& \leq \exp \left(-\gamma n^{1-5 \eta}\right) \text { if } h_{n} \geq n^{-\eta}
\end{aligned}
$$

On the other hand, the second condition in (19) guarantees that, for $n$ large enough, $n h_{n} v_{n}^{-2} \geq[2 /(\eta \gamma)] \log \left(1 / h_{n}\right)$, and thus

$$
\begin{aligned}
\exp \left(-\gamma \frac{n h_{n}}{v_{n}^{2}}\right) & \leq \exp \left(-\frac{2}{\eta} \log \left(1 / h_{n}\right)\right) \\
& \leq n^{-2} \text { if } h_{n} \leq n^{-\eta}
\end{aligned}
$$

The combination of (20) and (21) gives (18), which concludes the proof of Propositions 1 and 2, and of Corollaries 3 and 4.

\section{References}

Bahadur, R., 1960, On the asymptotic efficiency of tests and estimators, Sankhya 22, 229259.

Borovkov, A. and Mogulskii, A., 1992, Large deviations and testing of statistical hypotheses, Proc. Inst. Math. Russian Acad. Sci., Siberian Division 19. English transl.: Siberian Adv. Math., 2(3,4), 1992; 3(1,2), 1993.

Chernov, H., 1952. A measure of asymptotic efficiency for tests of a hypothesis based on the sum of observations, Ann. Math. Statist. 23, 497-507.

Davison, A.C. and Tsai, C.L., 1992. Regression model diagnostics, Internat. Statist. Rev. 60, 337-353.

Dembo, A. and Zeitouni, O., 1998. Large deviations techniques and applications. Springer. Applications of mathematics, New-York.

Dette, H. and Munk, A., 1998. A simple goodness-of-fit test for linear models under a random design assumption, Ann. Inst. Statist. Math. 50, 253-275.

Dette, H. and Munk, A., 1998. Validation of linear regression models, Ann. Statist. 26, 778-800.

Journal home page: www.jafristat.net; www.projecteuclid.org/as 
Dette, H., Munk, A. and Wagner, T., 1998. Estimating the variance in nonparametric regression - what is a reasonable choice? J. R. Stat. Soc. Ser. B Stat. Methodol. 60(4), 751-764.

Dieboldt, J., 1995, A nonparametric test for the regression function: asymptotic theory, $J$. Statist. Plann. Inference 44, 1-17.

Eubank, R.L. and Hart, J.D., 1992. Testing goodness of fit in regression via order selection criteria, Ann. Statist. 20, 1412-1425.

Eubank, R.L. and Spiegelmann, C.H. 1990, Testing the goodness of fit of a linear model via regression techniques, J. Amer. Statist. Assoc. 85, 387-392.

Gasser, T., Sroka, L. and Jennen-Steinmetz, C., 1986. Residual variance and residual pattern in nonlinear regression, Biometrika 73, 625-633.

Groeneboom, P., 1980. Large deviations and asymptotic efficiencies, Z. Mathematical Centre Tracts 118, Amsterdam.

Hall, P., 1992. Effect of bias estimation on coverage accuracy of bootstrap confidence intervals for a probability density, Ann. Statist. 20, 675-694.

Härdle, W. and Mammen, E., 1993. Comparing nonparametric versus parametric regression fits, Ann. Statist. 21, 1926-1947.

Härdle, W. and Marron, J.S., 1990. Semiparametric comparison of regression curves, Ann. Statist. 18, 83-89.

Ibragimov, I. and Radavicius, M., 1981. Probability of large deviations for the maximum likelihood estimator, Soviet. Math. Dokl. 23, 403-406.

Kallengerg, W., 1982. Chernoff efficiency and deficiency, Ann. Statist. 10, 583-594.

Kallengerg, W., 1983. Intermediate efficiency, theory and examples, Ann. Statist. 11, 170182.

Kallengerg, W., 1983. On moderate deviation theory in estimation, Ann. Statist. 11, 498-504.

Korostelev, A. and Leonov, S., 1995. Minimax Bahadur efficiency for small confidence intervals, Discussion paper 37, Humboldt University, Berlin

Mohdeb, Z. and Mokkadem, A., 2004. Average squared residuals approach for testing linear hypothesis in nonparametric regression, J. Nonparametric Stat. 16, 3-12.

Mohdeb, Z. and Mokkadem, A., 2015. Testing linear regression models in non regular case, Comm. Statist. Theory Methods 44, 4476-4490.

Mokkadem, A. and Pelletier, M., 2006. Confidence bands for densities, logarithmic point of view, Alea 2, 231-266.

Munk, A. and Dette, H., 1998. Nonparametric comparison of several regression functions: exact and asymptotic theory, Ann. Statist. 26, 2339-2368.

Nikitin, Y., 1995. Asymptotic efficiency of nonparametric tests, Cambridge University Press

Petrov, V.V., 1995. Limit theorems in probability theory, Clarendon Press, Oxford

Puhalskii, A. and Spokoiny, V., 1998. On large deviation efficiency in statistical inference, Bernoulli 4, 203-272.

Stadtmüller, U., 1986. Asymptotic properties of nonparametric curve estimates, Periodica Math. Hungar. Springer. 17, 83-108.

Stute, W., 1997. Nonparametric model checks for regression, Ann. Statist. 25, 613-641.

Worms, J., 2001. Moderate and large deviations of some dependent variables, Part II: some kernel estimators. Math. Methods Statist. 10(2), 161-193. 